\title{
Collapsing of glass tubes: analytic approaches in a hydrodynamic problem with free boundaries
}

\author{
Thomas Klupsch · Zhiwen Pan
}

Received: 5 January 2016 / Accepted: 28 January 2017 / Published online: 6 June 2017

(C) The Author(s) 2017. This article is an open access publication

\begin{abstract}
We present an analytic study to support the understanding of collapsing of glass tubes released by local heating and driven by the surface tension only. Our results provide a reliable analytic base for data evaluation if viscosity and surface tension of molten glasses are measured through collapsing. We complete existing 1D approaches to arrive at a consistent 2D description for axial symmetrical arrangements. We focus on advancing, steady-state collapsing profiles for heat sources moving with a constant axial velocity. The glass is considered an incompressible Stokes fluid with temperature-dependent viscosity of Arrhenius type that is ad hoc modeled by an axial, steady-state viscosity course comoving with the heat source. The analysis is carried out in comoving coordinates. Stringent scaling properties of collapsing are derived. We assume two distinct governing length scales $\tilde{L}$ and $\tilde{h}$ in axial and radial directions, respectively, as the base of a joint asymptotic multiscale analysis (AMSA) of the Stokes equation, boundary conditions, and kinematics for small $\tilde{h} / \tilde{L}$. Numerical studies of collapsingrelevant parameters in comparison with finite element reference calculations are outlined for axial courses of the reciprocal viscosity idealized as Gaussian. For arbitrary axial viscosity courses, AMSA results in a consistent analytical description of collapsing for small $\tilde{h} / \tilde{L}$. For $\tilde{h} / \tilde{L}<1$, precise formulae for collapsing-relevant geometry parameters are outlined.
\end{abstract}

Keywords 2D analytic solution - Asymptotic multiscale analysis - Collapsing of glass tubes · Free boundary kinematics · Variable viscosity

T. Klupsch $(\varangle)$

Department of Fiber Optics, Leibniz Institute of Photonic Technologies, Albert-Einstein-Strasse 9, 07745 Jena, Germany e-mail: thomas.klupsch@t-online.de

T. Klupsch

P.O. Box 100239, 07702 Jena, Germany

T. Klupsch

Mädertal 4, 07745 Jena, Germany

Present Address

Z. Pan

Otto-Schott-Institut für Glaschemie, Friedrich-Schiller-Universität Jena, 07743 Jena, Germany 


\section{Introduction}

When an axial section of a glass tube is heated such that the glass becomes a viscous fluid, the tube will collapse back upon itself inside the heating zone. This effect also occurs if the gravity is neglected, and neither an axial streaming beyond the heating zone nor any pressure difference between the hollow and the area beyond the tube exists. Then the surface tension is the only driving force. Such an elementary process is called collapsing (see, e.g., [1-8]). We will exclusively focus on collapsing with a uniformly moving heat source, where an observer comoving with the heat source becomes aware of a steady-state collapsing profile narrowed against the direction of motion [2-7]. Of course, this only occurs if the comoving observer measures, at the same time, an accompanying steady-state temperature field. Collapsing has to be distinguished from drawing, that is, the successive narrowing of glass tubes or rods up to capillary dimensions by passing through a heating zone where, in addition, different axial streaming velocities on either side of the heating zone are impressed (see, e.g., [9,10]). Then the impressed axial flow difference plays the role of the proper driving force, whereas the surface tension would not be really required to understand drawing of tubes with axial symmetry (see, e.g., [9]).

For clarity, the mathematical description chosen throughout in this paper will strictly adopt the arrangements for viscosity measurements through collapsing described in [2-4]. In particular, we will assume that the cold ends of the glass tube are fixed in the laboratory frame. Consequently, the viscous flow that is to be calculated is always considered to be measured in the laboratory frame. This also implies that beyond the heating zone, where the glass rapidly approaches the properties of a rigid body, the viscous flow tends to zero.

This paper is motivated by the concern to have a well-founded analytical base for data evaluation, if systematic and contactless measurements of viscosity and surface tension of molten glasses are carried out through collapsing with a moving heat source $[3,4]$. In addition, both collapsing and drawing are fundamental technological steps in manufacturing of optical fibers (see, e.g., [1-12]). Always for reasons of economy in time, conditions should be predicted to assure the fabrication of optical fibers where, at least, the desired properties are approached (see, e. g. $[12,13])$. Analysis of determining factors is a matter of the theory of analytic modeling, in particular. One of the most important premises is, of course, that the governing material parameters, in particular, the temperature-dependent viscosity and the surface tension of the special glasses, are known precisely enough. Vice versa, a correct modeling is the base to exploit collapsing and drawing to measure the material parameters in question (see, e.g., [3,4,8, 14]).

There is a large series of papers on analytic approaches of the drawing problem since the 1970s (e.g., $[9,10,14-$ 29]), but only fewer papers involve an analytic treatment of collapsing [1-8] since the 1980s. The approaches on drawing (axial symmetry assumed) favor a perturbation analysis with the prerequisite of a dominating axial viscous flow and axial viscous flow gradient, small axial viscosity dependence, and small inclination of the rod boundary (or tube boundaries) against the rotation axis, as typical for capillary geometries. This idea goes back, in principle, to Lord Rayleigh's early paper on the axial symmetric vena contracta problem [30] and has been continued to understand the spinning of textile fibers from polymers [31], and the drawing of glassy rods [10]. Also, the perturbation analysis has been proven to be rather powerful to include accompanying effects such as the stability (e.g., $[9,10,16,17])$, temperature-dependent viscosity (e.g., $[9,10,14,18,19])$, as well as heat conduction, convection, and radiation (e.g., $[9,10,15,16])$. Recently, it is applied to the drawing of hollow and structured fibers (e.g., [9,22,25-29]). A substantial analysis of the mathematical and physical concepts on the perturbation treatment of drawing of tubes is given in the extended paper of Fitt et al. [9]. The simplified description for capillary geometries developed in [9] has been refined [27-29] and proved to agree well with the experiments [27] and finite element (FEM) calculations [28]. It has also been shown that the description for capillary geometries [9] provides practical insights into the drawing of more complex multi-hole geometries [29]. Based, in addition, on an earlier leadingorder analysis for extensional flows in slender geometries [32], a mathematical leading-order framework of fiber drawing, specified to arbitrary cross-plane geometries, is applied in [33] and subsequent papers [34,35].

The known hydrodynamic approaches of collapsing [1-8] assume throughout a governing radial viscous flow, so that the collapsing kinematics is understood to result from a radial movement of the tube boundaries, driven by external forces in radial direction. Then the hydrodynamics reduce to the radial viscous flow and radial normal stress, both depending upon the radial coordinate only. In other words, the axial viscous flow gradient is ad hoc 
considered to be a small quantity that can be neglected within a leading-order treatment. This analysis will be denoted in the following as the 1D collapsing theory. A first extension toward a 2D analysis to study azimuthal collapsing instabilities of thick-walled tubes under stationary heat sources has been reported in [5,7]. Another 2D extension outlined in [6] takes into account the axial heat conduction to specify the axial and radial temperature and viscosity distribution for moving heat sources. We also note that the 1D approach of collapsing was taken as the base to estimate the viscosity of molten silica glass through observation of the collapsing kinetics for capillary geometries, where glass tube and heat source are fixed with the laboratory [8].

Although the 1D theory has been successfully applied in practice, its ad hoc assumptions are not well founded from a theoretical viewpoint. In particular, the former is inappropriate to understand influencing, axially dependent factors satisfactorily, which naturally accompany collapsing. Namely, this regards the axial course of both the viscosity and boundary profiles across the collapsing zone. The extension toward a consistent 2D theory of collapsing which takes into account these accompanying axial effects in a systematic way, resulting in feasible analytic formulae and error estimations, is the aim of this paper. Azimuthal effects will be excluded. In particular, we will show that the 1D approach of collapsing can be taken as the starting approach of an asymptotic multiscale analysis (AMSA) to perform a systematic study of corrections due to the axial dependence of viscosity and surface profiles.

Of course, the approaches used in the drawing theory are adjusted, first of all, to arrive at a basic understanding of influencing factors of the technical fiber drawing process, such as peculiarities of drawing of fibers with complicated cross-sections (see, e.g., [33-35]). The limiting case of equal, impressed axial velocities on both sides of the heating zone (collapsing limit), if mentioned at all [9,26,33], is considered being of minor interest, e.g., for preform fabrication (see, e.g.,[33]). Known papers on collapsing only are not cited in the papers on drawing. The constraints to measure glass viscosities through collapsing are quite distinct. The experimental arrangement is chosen as simply as possible, meanwhile an enhanced analytic effort is required to describe the experiment as precisely as possible, which naturally includes the analysis of validity limits and error estimations. The known papers on drawing are not focused on such a precondition. Compared with AMSA, the approaches on drawing are based on distinct perturbation ansatzes. The above-mentioned accompanying axial effects that cause 2D correction in the collapsing theory will be suppressed herein because they appear as effects of second perturbation order. Therefore, the 2D results of our paper cannot be reproduced herein. This may justify our intention to study the complex collapsing problem independently of the cited series of papers on drawing. The consistency of our mathematical approach, as necessary for practical applications, will also be supported by selected ab initio FEM calculations for prescribed parameters.

In this paper, we will exclusively focus on the hydrodynamic aspect of collapsing, namely on steady-state properties of a comoving collapsing region for heat sources moving in an axial direction. In comoving coordinates, the viscosity (and surface tension) will be assumed, prescribed as a steady-state function depending upon the axial coordinate only. To predict collapsing-relevant data in practice, the axial course of viscosity (and surface tension) must be back-calculated from their known temperature dependence, provided that the axial temperature course has been determined, e.g., from pyrometric measurements of the surface temperature (see, e.g., [36-39]), or from a theoretical analysis of the energy balance including heat conduction and thermal radiation (for the general aspect, see, e.g., [36]). Vice versa, viscosity (and surface tension) can be measured through back-calculation from experimental collapsing data, based on a fit of free coefficients entering a presupposed law of the viscosity temperature dependence. Such a treatment remains unsatisfactory in as far as the strong temperature dependence of the viscosity of glass (see, e.g., [39]) would require, in addition, a sufficiently precise determination of the temperature inside the tube wall, namely for temperatures up to $2500 \mathrm{~K}$ (see, e.g., the discussions for tubes with capillary dimensions in $[9,10,15,16,27,28])$. Recently, an extended study for tubes with wall thickness exceeding capillary dimensions has been given in [40], where the consequences for viscosity measurements through collapsing are discussed, too. To succeed in applications, the approaches favored in our paper may be restricted to tubes with a sufficiently small wall thickness, or extrapolations to zero wall thickness may be used. This problem is less exciting for the drawing of glass tubes with "optically thin" capillary dimensions and has been discussed in some papers on drawing cited above.

This paper is organized as follows: The underlying basic hydrodynamic equations and boundary conditions are outlined in Sect. 2, and specified to the rotation symmetric, steady-state collapsing problem for moving heat 
sources in the Stokes approximation, with the viscosity varying in axial direction. In addition, scaling properties are derived, and an appropriate reformulation of the Stokes equations is given there. In Sect. 3, the collapsing problem is treated applying the methods of the asymptotic multiscale analysis to the governing differential equations, boundary conditions, and kinematics, starting from the 1D theory as zeroth approach. Finally, in Sect. 4, the outlined analytical approaches of collapsing are discussed in summary and compared with numerical results from FEM.

\section{Principles of analytic modeling}

2.1 Coordinates, equations, and boundary conditions

Let $(r, \varphi, z)$ be a cylindrical coordinate system with $z$-axis as the rotation axis of the collapsing tube. Axial symmetry will be assumed throughout so that all physical quantities in question may depend only upon $r, z$, and time $t$. In particular, the viscous flow $\mathbf{v}(r, z, t)$ may be given as

$\mathbf{v}(r, z, t)=\hat{\mathbf{e}}_{r} \cdot v_{r}(r, z, t)+\hat{\mathbf{e}}_{z} \cdot v_{z}(r, z, t)$

where $\hat{\mathbf{e}}_{r}, \hat{\mathbf{e}}_{z}$ are the radial and axial unit vectors and $v_{r}(r, z, t), v_{z}(r, z, t)$ the radial and axial flow components, respectively. Molten glass will be considered to be incompressible, that is

$\nabla \cdot \mathbf{v}(r, z, t)=0$

$\eta(r, z, t) \cdot \sigma^{\prime}(r, z, t)$ denotes that part of the total stress tensor originated by the viscous material properties (see, e.g., [41], Chap. 2). $\eta(r, z, t)$ is the dynamic viscosity depending, in general, upon space and time, and $\boldsymbol{\sigma}^{\prime}$ is determined by the viscous flow only. For axial symmetry, there are five non-vanishing components of the symmetric tensor $\sigma^{\prime}$ in cylindrical coordinates, where we need $[41,42]$

$\sigma_{r r}^{\prime}=2 \partial v_{r} / \partial r$

$\sigma_{r z}^{\prime}=\sigma_{z r}^{\prime}=\partial v_{r} / \partial z+\partial v_{z} / \partial r$

$\sigma_{z z}^{\prime}=2 \partial v_{z} / \partial z$

whereas $\sigma_{\varphi \varphi}^{\prime}=2 v_{r} / r$ is unimportant in the following. Regarding the space dependence of $\eta$, the Navier-Stokes equation for incompressible fluids reads (see, e.g., [41,42])

$\rho(\partial \mathbf{v} / \partial t+(\mathbf{v} \cdot \nabla) \mathbf{v})=-\nabla p+\eta \Delta \mathbf{v}+\sigma^{\prime} \cdot \nabla \eta$,

where $\rho$ is the mass density of the glass and $p=p(r, z, t)$ is the hydrodynamic pressure related to the viscous flow. The gravity, as well as the centrifugal force in a rotating collapsing tube will be neglected. As mentioned above, the space and time dependence of $\eta$ is caused by its temperature dependence. The same holds true for $\rho$, but this is unimportant, regarding approaches introduced later. Below, we will show that the kinematics of the collapsing process, as well as the course of steady-state collapsing profiles is ultimately governed by the temperature dependence of $\eta$. It is accepted to describe the temperature dependence of the dynamic viscosity of glasses by Arrhenius relations (see, e.g., [3,39] and references cited therein). For silica, one may adopt, for a wide temperature range from about $1300 \mathrm{~K}$ up to $2500 \mathrm{~K}$, an Arrhenius fit ${ }^{1}$ according to

$\eta_{\mathrm{emp}}(T)=\exp (-18.77+66420 / T)$

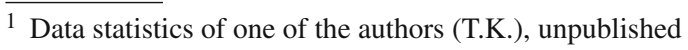


with $\eta_{\mathrm{emp}}(T)$ being the empirical, temperature-dependent dynamic viscosity (in $[\mathrm{Pa} \cdot \mathrm{s}]$ ) and $T$ the temperature (in [ K ]). There are other empirical Arrhenius-like viscosity relations for silica and other glasses (see, e.g., [3,39]), too, where, for silica, the logarithm of the former may differ from that of (2.7) in the ten percent range.

We will analyze steady-state collapsing induced by a heat source moving uniformly in $z$-direction with the velocity $v_{T}$. The mathematics become substantially simplified if we distinguish between the frame, where the flow components are measured, and coordinates on which the flow components depend. Precisely, (i) we measure the viscous flow in the laboratory system (see our remark at the beginning of Sect. 1); (ii) we change from laboratory coordinates $(r, z)$ to new coordinates $(\tilde{r}, \tilde{z})$ (such as "traveling wave coordinates"), which comove with the heat source, and then we have $\tilde{r}=r, \tilde{z}=z-v_{T} t$; and (iii) the viscous flow components measured in the laboratory system will be calculated as the functions of the comoving coordinates. A comoving observer may measure a steady-state axial temperature distribution. Regarding the temperature dependence of the viscosity given, e.g., by (2.7), a variable viscosity may be provided according to $\eta(z, t)=\tilde{\eta}(\tilde{z})$. Thus, it is suggested to look for solutions $\mathbf{v}(r, z, t)=\tilde{\mathbf{v}}(r, \tilde{z}, t), p(r, z, t)=\tilde{p}(r, \tilde{z}, t)$. The Navier-Stokes equation (2.6), if rewritten according to (i) to (iii), does not preserve its original form. Nevertheless, we can conclude, following the well-known argument [41,42], that the 1.h.s. is small, on the order of the Reynolds number $\operatorname{Re}=L U \bar{\rho} / \bar{\eta}$ compared with the r.h.s., where $L, \bar{\rho}$, and $\bar{\eta}$ stands for the order of the characteristic length scale (e.g., axial length of the heater), density, and viscosity of our problem, respectively, and $U$ is a characteristic velocity which now stands for $v_{T}$, too. For collapsing experiments, we have $L \approx 10^{-2} \mathrm{~m}, U \approx 10^{-5} \mathrm{~m} / \mathrm{s}$ (the order of $v_{T}$ ), $\bar{\rho} \approx 10^{3} \mathrm{~kg} / \mathrm{m}^{3}$, and $\bar{\eta} \approx 10^{4} \mathrm{~Pa}$ s (the order of the minimum viscosity for maximum temperatures of about $2300 \mathrm{~K}$ ), resulting in $\operatorname{Re} \approx 10^{-8}$. Therefore, the Stokes approximation (see, e.g., [41], Chap. 2) for highly viscous fluids can be applied, neglecting the 1.h.s. of (2.6) in comoving coordinates. The resulting Stokes equation [41,42] preserves, in fact, its form carrying out (i) to (iii). These findings are important from physical, as well as mathematical viewpoint and will be discussed below. In the same way, the definitions (2.3) to (2.5) of the components of $\sigma^{\prime}$ remain the same substituting $z$ by $\tilde{z}$.

Here we will make the first simplification of notation by denoting quantities in comoving coordinates with the axial coordinate denoted by $z$ (not $\tilde{z}$ ) (in case of doubt, the choice of the coordinate system will be outlined).

For incompressible fluids, the Stokes equations read

$$
\begin{aligned}
& \nabla \cdot \mathbf{v}=0, \\
& \eta \Delta \mathbf{v}+\sigma^{\prime} \cdot \nabla \eta=\nabla p .
\end{aligned}
$$

The steady-state viscous flow $\mathbf{v}(r, z)$ and pressure $p(r, z)$ must satisfy two groups of boundary conditions. The first one concerns the force balance at the surface of the collapsing glass tube and the second one the behavior of the viscous flow at the axial boundaries of the collapsing region. In the following, we will call them the radial boundary conditions $(\mathrm{RBC})$ and the axial boundary conditions $(\mathrm{ABC})$, respectively. According to the collapsing model introduced above, the RBC embrace the balance of the internal frictional force from the viscous flow and the external force due to the surface tension. The steady-state outer and inner surfaces of the collapsing glass tube according to (ii) may be represented by $r=R_{0}(z)$ and $r=R_{1}(z)$, respectively. The associated tangential and normal unit vectors may be denoted by $\mathbf{t}_{0}(z), \mathbf{t}_{1}(z)$, and $\mathbf{n}_{0}(z), \mathbf{n}_{1}(z)$, respectively, where both the normal vectors may be directed outward with respect to the collapsing glass tube. We have

$$
\begin{aligned}
& \mathbf{t}_{i}(z)=\hat{\mathbf{e}}_{r} \cdot \frac{\partial R_{i}(z) / \partial z}{\sqrt{1+\left(\partial R_{i}(z) / \partial z\right)^{2}}}+\hat{\mathbf{e}}_{z} \cdot \frac{1}{\sqrt{1+\left(\partial R_{i}(z) / \partial z\right)^{2}}}, \\
& \mathbf{n}_{i}(z)=(-1)^{i}\left(\hat{\mathbf{e}}_{r} \cdot \frac{1}{\sqrt{1+\left(\partial R_{i}(z) / \partial z\right)^{2}}}-\hat{\mathbf{e}}_{z} \cdot \frac{\partial R_{i}(z) / \partial z}{\sqrt{1+\left(\partial R_{i}(z) / \partial z\right)^{2}}}\right),
\end{aligned}
$$

where $i=0,1$ may indicate the outer and inner boundaries, respectively. The RBC can be decomposed into two groups of equations corresponding to the balance of the normal and tangential force components, respectively. The external force at the outer and inner surfaces is given by $-(-1)^{i} \cdot \mathbf{n}_{i}(z) \cdot 2 \tau H_{i}(z)$, respectively, where $\tau$ is the surface tension. For silica, $\tau$ is widely temperature independent for temperatures that are accessible for collapsing 
[3], but in general this is not true for other (doped) glasses [39]. In what follows, we will deal with constant $\tau$, for simplification only. We will show in the next section that analysis of the RBC can be straightforwardly extended if $\tau$ depends upon $z$ via its temperature dependence. $H_{i}(z)$ denotes the mean curvature of the outer and inner surfaces, respectively (see, e.g., [41], Chap. 7). Consequently, the external force enters the first group of the RBC only. For a surface of revolution given by $r=R(z)$ in cylindrical coordinates, the mean curvature is (see, e.g., [43])

$H(z)=\frac{1}{2 R(z)} \cdot \frac{1}{\sqrt{1+(\partial R(z) / \partial z)^{2}}}-\frac{\partial^{2} R(z) / \partial^{2} z}{{\sqrt{1+(\partial R(z) / \partial z)^{2}}}^{3}}$.

The first group of the RBC reads (see, e. g. [41], Chap. 2)

$\mathbf{n}_{i}(z) \cdot\left[p\left(R_{i}(z), z\right)-\eta(z) \boldsymbol{\sigma}^{\prime}\left(R_{i}(z), z\right)\right] \cdot \mathbf{n}_{i}(z)=(-1)^{i} \cdot 2 \tau H_{i}(z)$

$i=0,1$. If an additional hollow pressure $p_{h}$ is impressed to carry out a joint determination of viscosity and surface tension through two independent measurements (see, e.g., [3]), the term $p\left(R_{i}(z), z\right)$ in (2.13) for $i=1$ has to be substituted by $p\left(R_{i}(z), z\right)-p_{h}$. In the same way, an external torch pressure $p_{T}$ from streaming hot gases impinging the outer tube can be taken into account substituting $p\left(R_{i}(z), z\right)$ in (2.13) by $p\left(R_{i}(z), z\right)-p_{T}$ for $i=0$. The second group of RBC reads, observing $\mathbf{t}_{i}(z) \cdot \mathbf{n}_{i}(z)=0$ :

$\mathbf{t}_{i}(z) \cdot \boldsymbol{\sigma}^{\prime}\left(R_{i}(z), z\right) \cdot \mathbf{n}_{i}(z)=0$.

The ABC are simply written down according to (i)-(iii). We have the obvious physical condition that the viscous flow must become slowed down to zero if the glass approaches a rigid body outside the heating zone. Then the $\mathrm{ABC}$ for collapsing of an infinitely extended tube become

$\lim _{|z| \rightarrow \infty} \mathbf{v}(r, z, t)=0$

if $\lim _{|z| \rightarrow \infty} \eta(r, z, t)=\infty$.

Finally, the RBC and $\mathrm{ABC}$ have to be found by kinematic equations of the free boundaries, which implies that the movement of the free boundaries is governed, vice versa, by the viscous flow. For steady-state collapsing profiles in comoving coordinates, a simple geometrical consideration results in the two first-order ordinary differential equations:

$\mathrm{d} R_{i}(z) / \mathrm{d} z=-v_{r}\left(R_{i}(z), z\right) /\left[v_{T}-v_{z}\left(R_{i}(z), z\right)\right]$,

where $i=0,1$. These kinematic equations close the mathematical problem of steady-state collapsing.

\subsection{Dimensionless quantities, scaling, and basic aspects of an analytic treatment}

The dimensionless coordinates and geometric and hydrodynamic quantities introduced in the following are specified to collapsing and based on an unconventional velocity benchmark. This treatment is distinguished from that commonly used for drawing (see, e.g., [9,32]). In this section only, the dimensionless quantities are temporarily denoted with an overbar. The length will be measured in units of the benchmark $\Lambda$, specified below. Dimensionless cylindrical coordinates $(\bar{r}, \bar{\varphi}, \bar{z})$ are introduced via $\bar{r}=r / \Lambda, \bar{z}=z / \Lambda, \bar{\varphi}=\varphi$, and the steady-state dimensionless outer and inner profile functions in moving, dimensionless cylindrical coordinates are denoted by $\bar{r}=\bar{R}_{0}(\bar{z})$ and $\bar{r}=\bar{R}_{1}(\bar{z})$, respectively. The corresponding tangential and normal unit vectors and the dimensionless curvature $\bar{H}(\bar{z})$ are found by applying (2.10), (2.11), and (2.12) in dimensionless coordinates, respectively, where 
$H(z)=(1 / \Lambda) \cdot \bar{H}(\bar{z})$. The axial coordinate $z$ will be specified such that the measured temperature distribution $T(z)$, and the viscosity $\eta(z)$ calculated from $T(z)$ assumes the maximum $T_{\max }$ and minimum $\eta_{\min }$, respectively, at $z=0$. We introduce dimensionless viscosity $\bar{\eta}$, viscous flow $\overline{\mathbf{v}}$, pressure $\bar{p}$, and tensor $\overline{\boldsymbol{\sigma}}^{\prime}$, all depending upon the dimensionless coordinates, by $\bar{\eta}(\bar{z})=\eta(\Lambda \bar{z}) / \eta_{\min }, \overline{\mathbf{v}}(\bar{r}, \bar{z})=\left(\eta_{\min } / \tau\right) \cdot \mathbf{v}(\Lambda \bar{r}, \Lambda \bar{z}), \bar{p}(\bar{r}, \bar{z})=(\Lambda / \tau) \cdot p(\Lambda \bar{r}, \Lambda \bar{z})$, and $\bar{\sigma}^{\prime}(\bar{r}, \bar{z})=\left(\Lambda \cdot \eta_{\min } / \tau\right) \sigma^{\prime}(\Lambda \bar{r}, \Lambda \bar{z})$. The dimensionless velocity $\bar{u}$ of the heat source is $\bar{u}=\left(\eta_{\min } / \tau\right) \cdot v_{T}$, and the dimensionless time is $\bar{t}=t \cdot \tau /\left(\Lambda \cdot \eta_{\min }\right)$. The velocity benchmark $\tau / \eta_{\min }$ is the capillary velocity at the maximum temperature.

The hydrodynamic equations (2.8), (2.9), the RBC (2.13), (2.14), and the kinematic equations (2.16), if rewritten in dimensionless coordinates and quantities, are invariant against the transformation to dimensionless coordinates and quantities defined above, with the formal substitution $\tau=1$. This invariance means that all physical quantities entering the collapsing problem become scaled by the three basic parameters $\Lambda, \eta_{\min }$, and $\tau$. In particular, any quantities $x, t, v$, and $p$ with dimensions of length, time, velocity, and pressure related to the corresponding dimensionless ones by $x=\Lambda \bar{x}, t=\left(\eta_{\min } \Lambda / \tau\right) \cdot \bar{t}, v=\left(\tau / \eta_{\min }\right) \cdot \bar{v}$, and $p=(\tau / \Lambda) \cdot \bar{p}$. Any quantities $\bar{x}$, $\bar{t}, \bar{v}$, and $\bar{p}$ thought to result from the dimensionless analysis of steady-state collapsing will be determined by the dimensionless tube geometry at the start, the course $\bar{\eta}(\bar{z})$ of the dimensionless viscosity, and the dimensionless velocity $\bar{u}$ of the heat source only.

If the temperature dependence of the surface tension cannot be neglected, we have to take into account a steadystate $z$-dependent surface tension $\breve{\tau}(z)$, such as the $z$-dependent viscosity. The dimensional parameter $\tau$ may be defined, at the beginning, as the surface tension at the maximum temperature $T=T_{\max }$, that is $\tau=\breve{\tau}(0)$. We introduce, like the dimensionless viscosity $\bar{\eta}$, the dimensionless surface tension $\bar{\tau}$ as $\bar{\tau}(\bar{z})=\breve{\tau}(\Lambda z) / \tau$. Then $\bar{\tau}(\bar{z})$ appears as a factor on the r.h.s. of the non-dimensionalized RBC (2.13).

In the following, we will make the second simplification of notation that, starting from $(2.8),(2.9)$ together with (2.13) to (2.16), we may set $\tau=1$ and denote all dimensionless quantities by omitting the overbar.

It is worth discussing some basic aspects to succeed in the analytic modeling of collapsing. Physically speaking, the Stokes flow does not exhibit any intrinsic dynamics and responds to any time-dependent change of the boundary conditions (surface profiles) without delay. This is the prerequisite that the kinematics of surface profiles become decoupled from the hydrodynamics. In fact, assuming a solution manifold $\mathbf{v}=\mathbf{v}\left(r, z,\left\{R_{0}, R_{1}\right\}\right)$, $p=p\left(r, z,\left\{R_{0}, R_{1}\right\}\right)$ of the Stokes equations (2.8), (2.9) and the RBC and ABC would be known, including the functional dependence upon any arbitrary boundary courses $R_{0}(z), R_{1}(z)$. We would arrive, after substitution of $\mathbf{v}=\mathbf{v}\left(r, z,\left\{R_{0}, R_{1}\right\}\right)$ into (2.16), at autonomous kinematic equations, which would cover the entity of steadystate collapsing profiles. Thus, the governing step to succeed in analytic approaches of collapsing is to establish appropriate approaches of hydrodynamic quantities for appropriately prescribed boundary profiles.

This aim requires further advanced ideas for an obvious modeling because the viscous flow will be determined, in general, to depend in a non-local way upon the entire course of $\eta(z), R_{0}(z)$ and $R_{1}(z)$, whereas this knowledge would be required for arbitrary courses of $R_{0}(z)$, and $R_{1}(z)$. One way to succeed is to look for local approaches where the viscous flow $\mathbf{v}(r, z)$ at the axial position $z$ becomes determined by $\eta(z), R_{0}(z), R_{1}(z)$, and local derivatives at the same $z$-position only. If existing, such a local approach implies the elimination of hydrodynamics in favor of kinematics, which leads to a strong mathematical simplification. Namely, the substitution of $\mathbf{v}(r, z)$ into the steadystate kinematic equations (2.16) would result in a closed system of ordinary differential equations to determine the steady-state profiles $R_{0}(z), R_{1}(z)$ for a moving heat source.

The leading idea to arrive at such a local description in the theory of collapsing, like in the drawing theory [9], is the assumption of two different governing length scales $\tilde{L}$ and $\tilde{h}$, which stand for characteristic axial and radial lengths (e.g., length of the heating zone and maximum tube diameter), respectively, where $\tilde{h} / \tilde{L} \ll 1$. The 1D theory of collapsing characterized the above changes for $\tilde{h} / \tilde{L} \rightarrow 0$ straightforwardly into a highly symmetric limiting case, which can be exactly solved. This is the base to establish the more complex asymptotic multiscale analysis (AMSA) of Sect. 3, to arrive at a local 2D theory of collapsing. In particular, the 1D theory of collapsing is derived in leading AMSA order, meanwhile the 2D description requires approaches beyond the leading order. This AMSA treatment is unconventional insofar as the flow field is now calculated depending on local derivatives of $R_{0}(z)$ and $R_{1}(z)$, too, so that the kinematic equations (2.16) become implicit differential equations. This circum- 
stance causes further restrictions beyond the basic AMSA preconditions, as shown in Sect. 3.5 in detail. Finally, it should be mentioned that, in principle, the AMSA treatment could be straightforwardly extended to drawing by providing for an additional leading-order axial flow with non-zero axial gradient across the heating zone only, together with the additional radial flow induced through the condition of incompressibility, and modified $\mathrm{ABC}$ and RBC. As always concluded (see, e.g., [9]), the leading-order AMSA shows that the drawing phenomenon is explainable without consideration of the surface tension. We will not study more details, which are out of our topic.

\subsection{The Stokes equations reformulated}

As emphasized in Sect. 1, we want to demonstrate an analysis of collapsing beyond the leading order. Therefore, we will perform an unconventional analysis of the Stokes equations (2.8), (2.9) for incompressible fluids with a space-dependent viscosity. Our aim, in particular, is (i) to eliminate (2.8) strictly to satisfy the incompressibility condition within each perturbation order, if AMSA is applied, and (ii) to derive the starting equations allowing a convenient way to deal with the space-dependent viscosity, namely $\eta=\eta(z)$. To eliminate (2.8), we introduce the vector field $\mathbf{A}(r, z)$ via

$$
\begin{aligned}
\mathbf{v}(r, z) & =\nabla \times \mathbf{A}(r, z), \\
\mathbf{A}(r, z) & =\hat{\mathbf{e}}_{\varphi} \cdot A_{\varphi}(r, z), \\
v_{r}(r, z) & =-\left(\frac{\partial}{\partial z}\right) A_{\varphi}(r, z), \\
v_{z}(r, z) & =\left(\frac{1}{r}\right)\left(\frac{\partial}{\partial r}\right)\left(r A_{\varphi}(r, z)\right)
\end{aligned}
$$

where $\hat{\mathbf{e}}_{\varphi}$ is the azimuthal unit vector. Thus, $A_{\varphi}(r, z)$ appears as a streaming function for rotational symmetric problems with an incompressible flow. Physically speaking, $\mathbf{A}(r, z)$ is the vector potential belonging to the incompressible viscous flow, as shown in Appendix 1.

Instead of (2.9), we change to coupled equations to calculate $A_{\varphi}(r, z)$ together with a pressure-related function $Q^{\prime \prime}(r, z)$. The resulting equations derived in Appendix 1 are

$$
\begin{aligned}
& \left(\Delta-\frac{1}{r^{2}}\right) Q^{\prime \prime}(r, z)=2 \cdot \frac{\mathrm{d}^{2} \eta(z)}{\mathrm{d} z^{2}} \cdot \frac{\partial}{\partial r} \frac{1}{r} \frac{\partial}{\partial r}\left(r A_{\varphi}(r, z)\right), \\
& \left(\Delta-\frac{1}{r^{2}}\right) A_{\varphi}(r, z)=\frac{Q^{\prime \prime}(r, z)}{\eta(z)}, \\
& p(r, z)=\frac{\partial Q(r, z)}{\partial z}+2 \frac{\mathrm{d} \eta(z)}{\mathrm{d} z} \frac{1}{r} \frac{\partial}{\partial r}\left(r A_{\varphi}(r, z)\right),
\end{aligned}
$$

where

$$
Q(r, z)=-\int^{r} \mathrm{~d} r_{1} Q^{\prime \prime}\left(r_{1}, z\right)+p_{0} z
$$

and $p_{0}$ is an arbitrary constant.

Equation (2.22) is simply understood. It relates the viscous flow through $A_{\varphi}(r, z)$ to the driving force through $Q^{\prime \prime}(r, z)$ with $1 / \eta$ being the kinetic coefficient. For illustration, we may choose $\eta(z)=$ const and consider the simple solution $Q^{\prime \prime}(r, z)=C r, A_{\varphi}(r, z)=(C / 4 \eta)\left(r^{3} / 2-R^{2} r\right)$ of (2.21), (2.22) where $C$ is an arbitrary constant. We find $\partial p / \partial r=0, \partial p / \partial z=2 C$ from (5.6), and $v_{r}(r, z)=0, v_{z}(r, z)=(C / 2 \eta)\left(r^{2}-R^{2}\right)$ from (2.19), (2.20). This is the well-known laminar axial flow within a tube of radius $R$ in $+z$-direction for $\partial p / \partial z<0$ (see, e.g., $[41,42])$, which satisfies the no-slip condition $v_{z}(r, z)=0$ at $r=R$. 


\section{Asymptotic multiscale analysis of collapsing}

\subsection{Principles and 1D theory revisited}

The principles of the asymptotic multiscale analysis (AMSA) as used in this work are outlined, e.g., in [44], Chap. 10. Our method is similar to Adomian's decomposition method [45] applied to linear partial differential equations with variable coefficients. First, we will extend AMSA to perform a joint analysis of the Stokes equations and boundary conditions, to construct the local approach of Sect. 2.2 for steady-state $R_{0}(z)$ and $R_{1}(z)$ in comoving coordinates. The kinematics will be discussed later. $R_{0 \max }, R_{0 \min }$ and $R_{1 \max }, R_{1 \min }$ may be the outer and inner tube radii before and after collapsing, respectively. We consider an infinitely extended axial region so that $R_{0 \max }=\lim _{z \rightarrow \infty} R_{0}(z)$, $R_{0 \min }=\lim _{z \rightarrow-\infty} R_{0}(z)$, and so on. The dimensionless units of Sect. 2.2 will be specified choosing $\Lambda=R_{0 \max }$. Occasionally, the outer and inner tube radii will also be denoted by $R_{0}^{(0)}$ and $R_{1}^{(0)}$, respectively.

The AMSA given here exploits the two governing length scales $\tilde{L}$ and $\tilde{h}$, as discussed in Sect. 2.2 (for further specification, see Sect. 3.5.2). The AMSA will be based on the exact solution of hydrodynamic equations and boundary conditions for the limiting case $\tilde{h} / \tilde{L} \rightarrow 0$. This implies the knowledge of the dependence upon parameters entering this problem. AMSA aims to construct solutions where relevant parameters become "slowly" $z$-dependent on the length scale $\tilde{L}$, with the condition to turn into the known solution for $\tilde{h} / \tilde{L} \rightarrow 0$. A trivial approach for sufficiently small $\tilde{h} / \tilde{L}$ would leave this known solution, but taking the parameters in question to become slowly $z$-dependent. Even, this is the choice of the zeroth-order AMSA. Subsequent corrections are found through an "entangled" perturbation analysis in powers of $\tilde{h} / \tilde{L}$, which shows a characteristic interplay between perturbing effects from differential equations and boundary conditions (see Sect. 3.3 and Appendix 2).

The 1D theory [2] corresponds to the starting approach of AMSA for $\tilde{L} \rightarrow \infty, \tilde{h} / \tilde{L} \rightarrow 0$. Then the ABC have to be replaced by symmetry conditions of invariance against translations and inversions. This implies $\eta(z)=\eta=1$, $v_{r}(r, z)=v_{r}, v_{z}(r, z)=0, p(r, z)=p(r), R_{0}(z)=R_{0}^{(0)}=$ const, and $R_{1}(z)=R_{1}^{(0)}=$ const (where $\eta$ will be explicitly outlined for reasons given below). There is one solution set of (2.21) to (2.24) satisfying these symmetry conditions, namely $Q^{\prime \prime}(r, z)=0, A_{\varphi}(r, z)=c_{A} \cdot z / r$, and $p(r, z)=c_{P}=$ const, with two arbitrary coefficients $c_{A}, c_{P}$. For symmetry reasons, the normal and tangential vectors at the boundaries are in radial and axial directions, respectively. Thus, the balance of the tangential (axial) force components (2.14) is satisfied because of $\sigma_{r z}^{\prime}=0$. Then $c_{A}, c_{P}$ are determined from the balance of the normal (radial) forces (2.13) of the RBC only. $v_{r}$ and $\sigma_{r r}^{\prime}$ are calculated using (2.19) and (2.3):

$$
\begin{aligned}
v_{r}(r) & =-c_{A} / r, \\
\sigma_{r r}^{\prime}(r, z) & =\sigma_{r r}^{\prime}(r)=2 c_{A} / r^{2},
\end{aligned}
$$

so that the radial force balance on both the boundaries $r=R_{0}^{(0)}, r=R_{1}^{(0)}$ becomes

$p(r, z) / \eta-\sigma_{r r}^{\prime}(r, z)= \pm(1 / \eta) \cdot(1 / r)$

with the lower sign to be taken for $r=R_{1}^{(0)}$. Then we find

$c_{A}=(1 / 2 \eta) \cdot R_{0}^{(0)} R_{1}^{(0)} /\left(R_{0}^{(0)}-R_{1}^{(0)}\right)$,

$c_{P}=1 /\left(\left(R_{0}^{(0)}-R_{1}^{(0)}\right)\right.$.

\subsection{Hydrodynamic functions and equations in AMSA}

The relevant parameters of the $1 \mathrm{D}$ theory are $\eta, R_{0}^{(0)}$, and $R_{1}^{(0)}$, to be considered to vary in axial direction on the length scale $\tilde{L} \gg \tilde{h}$. To underline this slow axial dependence, we introduce the formal and non-specified perturbation parameter $\varepsilon$, considered to be small, on the order of $\tilde{h} / \tilde{L} \ll 1$, and the "slow" axial variable $Z=\varepsilon z$, 
writing $\eta=\eta(Z), R_{0}=R_{0}(Z), R_{1}=R_{1}(Z)$. Then $\mathrm{d} \eta(z) / \mathrm{d} z=\varepsilon \mathrm{d} \eta(Z) / \mathrm{d} Z, \mathrm{~d} R_{0}(z) / \mathrm{d} z=\varepsilon \mathrm{d} R_{0}(Z) / \mathrm{d} Z$, and $\mathrm{d} R_{1}(z) / \mathrm{d} z=\varepsilon \mathrm{d} R_{1}(Z) / \mathrm{d} Z$. The terms $\mathrm{d} \eta(Z) / \mathrm{d} Z$ and so on with $\varepsilon$ as a prefactor are now $O(1)$. The formal perturbation parameter $\varepsilon$ is required to systematize the perturbation analysis. In the following, $Z$ and $\varepsilon$ will be outlined as arguments of functions we are looking for, writing $\overline{\bar{Q}}^{\prime \prime}(r, z, Z, \varepsilon), \overline{\bar{A}}_{\varphi}(r, z, Z, \varepsilon)$, and $\overline{\bar{p}}(r, z, Z, \varepsilon)$ instead of $Q^{\prime \prime}(r, z), A_{\varphi}(r, z)$, and $p(r, z)$, and so on. Then in the basic equations (2.21), (2.22), and (5.8), (2.23), the substitution

$$
\partial / \partial z \rightarrow \partial / \partial z+\varepsilon \cdot \partial / \partial Z
$$

must be carried out. Upon completion of the perturbation analysis, we may set $\varepsilon=1$ and verify that the terms in $\varepsilon^{n}$ become quantities of the order $(\tilde{h} / \tilde{L})^{n}$ due to their concrete parameter dependence.

To solve the differential equations, which have been brought in AMSA form in this way, $Z$ is formally treated as a free parameter, and all $Z$-derivatives may be collected on the r.h.s., so that the order in $\varepsilon$ of the r.h.s. is enhanced compared with the 1.h.s. Looking for solutions $\overline{\bar{Q}}^{\prime \prime}(r, z, Z, \varepsilon), \overline{\bar{A}}_{\varphi}(r, z, Z, \varepsilon)$ as perturbation series in $\varepsilon$, the idea is that the error due to the incomplete treatment of the axial dependence in lower perturbation orders becomes corrected in subsequent perturbation orders.

In the following, we present a strict AMSA version, assuming that any axial dependence is a slow one, suppressing the rapid axial variable $z$. Then we arrive at a rapidly proceeding perturbation analysis of the differential equations and boundary conditions in powers of $\varepsilon^{2}$. (2.21), (2.22) now read in AMSA version

$$
\begin{aligned}
& \frac{\partial}{\partial r} \frac{1}{r} \frac{\partial}{\partial r}\left(r \overline{\bar{Q}}^{\prime \prime}(r, Z, \varepsilon)\right)=-\varepsilon^{2} \frac{\partial^{2}}{\partial Z^{2}} \overline{\bar{Q}}^{\prime \prime}(r, Z, \varepsilon)+2 \varepsilon^{2} \frac{\mathrm{d}^{2} \eta(Z)}{\mathrm{d} Z^{2}}\left(\overline{\bar{Q}}^{\prime \prime}(r, Z, \varepsilon) / \eta(Z)-\varepsilon^{2} \frac{\partial^{2}}{\partial Z^{2}} \overline{\bar{A}}_{\varphi}(r, Z, \varepsilon)\right), \\
& \frac{\partial}{\partial r} \frac{1}{r} \frac{\partial}{\partial r}\left(r \overline{\bar{A}}_{\varphi}(r, Z, \varepsilon)-\overline{\bar{Q}}^{\prime \prime}(r, Z, \varepsilon) / \eta(Z)\right)=-\varepsilon^{2} \frac{\partial^{2}}{\partial Z^{2}} \overline{\bar{A}}_{\varphi}(r, Z, \varepsilon) .
\end{aligned}
$$

Equations (3.7), (3.8) are supplied by the AMSA version of (2.23) to determine the pressure $\overline{\bar{p}}(r, Z, \varepsilon)$ from known $\overline{\bar{Q}}^{\prime \prime}, \overline{\bar{A}}_{\varphi}$ according to

$\overline{\bar{p}}(r, Z, \varepsilon)=-\varepsilon \cdot \int^{r} \mathrm{~d} r_{1} \frac{\partial}{\partial Z} \overline{\bar{Q}}^{\prime \prime}(r, Z, \varepsilon)+2 \varepsilon \cdot \frac{\mathrm{d} \eta(Z)}{\mathrm{d} Z} \frac{\partial}{\partial r} \frac{1}{r} \frac{\partial}{\partial r} r \overline{\bar{A}}_{\varphi}(r, Z, \varepsilon)$.

We will solve (3.7), (3.8) by series expansions according to

$\overline{\bar{Q}}^{\prime \prime}(r, Z, \varepsilon)=\varepsilon \overline{\bar{Q}}^{\prime \prime(1)}(r, Z)+\varepsilon^{3} \overline{\bar{Q}}^{\prime \prime(3)}(r, Z)+\cdots$,

$\overline{\bar{A}}_{\varphi}(r, Z, \varepsilon)=(1 / \varepsilon) \cdot \overline{\bar{A}}_{\varphi}^{(-1)}(r, Z)+\varepsilon \cdot \overline{\bar{A}}_{\varphi}^{(1)}(r, Z)+\varepsilon^{3} \cdot \overline{\bar{A}}_{\varphi}^{(3)}(r, Z)+\cdots$.

After substitution of (3.10), (3.11) into (3.7), (3.8), the comparison in powers of $\varepsilon$ results in a chain of coupled ordinary differential equations with respect to $r$ only, to determine the higher-order terms of (3.10), (3.11) from the lowest order ones. Further hydrodynamic quantities in AMSA version can be calculated as power series in $\varepsilon$ from (3.9) to (3.11), applying (2.19), (2.20), and (2.3) to (2.5), with observing (3.6). In particular, the series expansions for the pressure, and the $r r$ - and $r z$-component of the stress tensor (divided by $\eta(Z)$ ) read

$$
\begin{aligned}
\overline{\bar{p}}(r, Z, \varepsilon) & =\overline{\bar{p}}^{(0)}(r, Z)+\varepsilon^{2} \overline{\bar{p}}^{(2)}(r, Z)+\varepsilon^{4} \overline{\bar{p}}^{(4)}(r, Z)+\cdots, \\
\overline{\bar{\sigma}}_{r r}^{\prime}(r, Z, \varepsilon) & =\overline{\bar{\sigma}}_{r r}^{\prime(0)}(r, Z)+\varepsilon^{2} \overline{\bar{\sigma}}_{r r}^{(2)}(r, Z)+\varepsilon^{4} \overline{\bar{\sigma}}_{r r}^{(4)}(r, Z)+\cdots, \\
\overline{\bar{\sigma}}_{r z}^{\prime}(r, Z, \varepsilon) & =\varepsilon \overline{\bar{\sigma}}_{r z}^{(1)}(r, Z)+\varepsilon^{3} \overline{\bar{\sigma}}_{r z}^{(3)}(r, Z)+\cdots
\end{aligned}
$$

(in (3.14), the term $\propto 1 / \varepsilon$ vanishes according to (5.13), (5.16) given below). 


\subsection{Boundary conditions in AMSA}

In AMSA, the ABC for an infinitely extended axial region become satisfied in an elementary way. We provide throughout that $1 / \eta(Z)$ is represented as exponential $1 / \eta(Z)=\exp (-f(Z))$, where $f(Z) \geq 0$, with maximum $1 / \eta(0)=1$ at $Z=0 . f(Z)$ may be an at least second-order polynomial $f(Z)=f_{2} Z^{2}+f_{3} Z^{3}+\cdots, f_{2}>0$, with $f(Z) \rightarrow \infty$ for $Z \rightarrow \pm \infty$. If the flow derived from the lowest order term in (3.11) satisfies the $\mathrm{ABC}$ for $Z \rightarrow \pm \infty$, this holds true in each perturbation order. These mathematical findings are simply verified from the perturbation analysis of (3.7), (3.8), noting that the exponential in question, if entering into the lowest order approach of $\overline{\bar{A}}_{\varphi}$, becomes reproduced in each perturbation order and in all quantities derived from $\overline{\bar{A}}_{\varphi}$. Our prerequisite regarding the viscosity course is physically motivated through the temperature dependence of the glass viscosity of Arrhenius type, like (2.7), and the characteristic axial temperature course across the collapsing region, where the temperature maximum is surrounded by steeply descending edges. Thus, AMSA naturally describes the suppression of the viscous flow beyond the heating zone for the preconditions outlined above.

The analysis of the $Z$-dependent $\mathrm{RBC}$ is a much more complex task. First, we will make the agreement that in this and subsequent sections, $R$ stands for $R_{0}(Z)$ and $R_{1}(Z)$, respectively. Our starting assumption is that any solution pair $\overline{\bar{Q}}^{\prime \prime}(r, Z, \varepsilon), \overline{\bar{A}}_{\varphi}(r, Z, \varepsilon)$ of (3.7), (3.8) satisfying the RBC, as well as the hydrodynamic functions derived from the former can be represented like the series expansions (3.10), (3.11), and (3.12) to (3.14), respectively. The 1.h.s. of (2.13) and (2.14), divided by $\eta(Z)$, may be abbreviated as

$$
\begin{aligned}
\overline{\bar{F}}_{\text {norm }}(R, Z, \varepsilon) & =\overline{\overline{\mathbf{n}}}(R, Z, \varepsilon) \cdot\left[\overline{\bar{p}}(R, Z, \varepsilon) / \eta(Z)-\overline{\bar{\sigma}}^{\prime}(R, Z, \varepsilon)\right] \cdot \overline{\overline{\mathbf{n}}}(R, Z, \varepsilon), \\
\overline{\bar{F}}_{\tan }(R, Z, \varepsilon) & =-\overline{\overline{\mathbf{t}}}(R, Z, \varepsilon) \cdot \overline{\bar{\sigma}}^{\prime}(R, Z, \varepsilon) \cdot \overline{\overline{\mathbf{n}}}(R, Z, \varepsilon)
\end{aligned}
$$

with $\overline{\overline{\mathbf{n}}}$ and $\overline{\overline{\mathbf{t}}}$ being the axial courses of the normal and tangential vectors at the boundaries, respectively. Then the series expansions of (3.15), (3.16) are found through expanding (2.10), (2.11) in powers of $\varepsilon$, with observing (3.6). In the same way, the mean curvature at the boundaries given by (2.12) will be written as $H(z)=\overline{\bar{H}}(R, Z, \varepsilon)$. We arrive at expansions in even and odd powers of $\varepsilon$ only, according to

$$
\begin{aligned}
\overline{\bar{F}}_{\text {norm }}(R, Z, \varepsilon) & =\overline{\bar{F}}_{\text {norm }}^{(0)}(R, Z)+\varepsilon^{2} \overline{\bar{F}}_{\text {norm }}^{(2)}(R, Z)+\varepsilon^{4} \overline{\bar{F}}_{\text {norm }}^{(4)}(R, Z)+\cdots, \\
\overline{\bar{F}}_{\text {tan }}(R, Z, \varepsilon) & =\varepsilon \overline{\bar{F}}_{\tan }^{(1)}(R, Z)+\varepsilon^{3} \overline{\bar{F}}_{\tan }^{(3}(R, Z)+\cdots, \\
\overline{\bar{H}}(R, Z, \varepsilon) & =\overline{\bar{H}}^{(0)}(R, Z)+\varepsilon^{2} \overline{\bar{H}}^{(2)}(R, Z)+\varepsilon^{4} \overline{\bar{H}}^{(4)}(R, Z)+\cdots .
\end{aligned}
$$

The conditions (2.13) and (2.14) of the normal and tangential force balance, respectively, at the boundaries can now be rewritten according to

$\overline{\bar{F}}_{\text {norm }}(R, Z, \varepsilon)= \pm 2 \overline{\bar{H}}(R, Z, \varepsilon) / \eta(Z)$,

$\overline{\bar{F}}_{\tan }(R, Z, \varepsilon)=0$

with the lower sign in (3.20) to be taken for $R=R_{1}(Z)$. After substitution of (3.17) to (3.19) into (3.20), (3.21), we see that (i) the RBC become satisfied through the normal force balance only in leading order $\varepsilon^{0}$, and (ii) in addition, through the tangential force balance only in $\varepsilon^{1}$, beyond the leading order. Continuing, contributions to the normal and tangential force balance will appear in higher even and odd powers of $\varepsilon$ only, respectively.

The AMSA treatment of the RBC implies, as already emphasized in Sects. 3.1 and 3.2, that any force balance condition in $\varepsilon^{n}$ will be satisfied with respect to the rapid (radial) variable only, meanwhile its $Z$-dependence is taken into account through $Z$-dependent coefficients. The resulting error will be corrected in two steps: (i) to arrive at the solutions of (3.7), (3.8), this $Z$-dependence implies corrections in subsequent orders, as described below (3.10), (3.11); and (ii) these corrections on their part will induce additional higher-order contributions to $\overline{\bar{F}}_{\text {norm }}(R, Z, \varepsilon)$ and $\overline{\bar{F}}_{\tan }(R, Z, \varepsilon)$, to be taken into account in the corresponding higher-order force balance conditions. Details are given in Appendix 2. 
As a result, the outlined quantities (3.10) to (3.16) as the solutions of (3.7), (3.8) and RBC can be represented, due to the successive settlement of the $\mathrm{RBC}$ in $\varepsilon^{0}, \varepsilon^{1}, \ldots$, as series, e.g.,

$\overline{\bar{A}}_{\varphi}(r, Z, \varepsilon)=\overline{\bar{A}}_{\varphi 0}(r, Z, \varepsilon)+\overline{\bar{A}}_{\varphi 1}(r, Z, \varepsilon)+\cdots$,

where $\overline{\bar{A}}_{\varphi 0}=O(1 / \varepsilon), \overline{\bar{A}}_{\varphi 1}=O(\varepsilon)$. These series could be rearranged to proceed strictly in powers of $\varepsilon$, which will not be done here.

\subsection{The viscous flow in the zeroth and first orders}

We will summarize the zeroth- and first-order results for the viscous flow and refer, for details, to Appendix 3. From general AMSA, the radial flow $\overline{\bar{v}}_{r}(r, Z, \varepsilon)$ and axial flow $\overline{\bar{v}}_{z}(r, Z, \varepsilon)$ result as series in even and odd powers of $\varepsilon$, respectively, observing that in the lowest order $\overline{\bar{A}}_{\varphi 0} \propto(1 / \varepsilon) \cdot(1 / r)$. For simplification, we will write in the following

$\overline{\bar{v}}_{r}(r, Z, \varepsilon)=\overline{\bar{v}}_{r 0}(r, Z)+\varepsilon^{2} \overline{\bar{v}}_{r 2}(r, Z)+\cdots$,

$\overline{\bar{v}}_{z}(r, Z, \varepsilon)=\varepsilon \overline{\bar{v}}_{z 1}(r, Z)+\varepsilon^{3} \overline{\bar{v}}_{z 3}(r, Z)+\cdots$.

From the zeroth- and first-order AMSA, we have

$$
\begin{aligned}
\overline{\bar{v}}_{r}(r, Z, \varepsilon)= & -\frac{1}{2 \eta(Z)} \cdot \frac{R_{0}(Z) R_{1}(Z)}{R_{0}(Z)-R_{1}(Z)} \cdot \frac{1}{r}+O\left(\varepsilon^{2}\right), \\
\overline{\bar{v}}_{z}(r, Z, \varepsilon)= & \varepsilon \frac{\mathrm{d} R_{0}(Z)}{\mathrm{d} Z} \frac{1}{2 \eta(Z)} \cdot \frac{R_{1}(Z)}{R_{0}(Z)^{2}-R_{1}(Z)^{2}} \cdot\left(\frac{r^{2}}{R_{0}(Z)-R_{1}(Z)}-\frac{R_{1}(Z) \cdot(1+2 \log r)}{2}\right) \\
& +\varepsilon \frac{\mathrm{d} R_{1}(Z)}{\mathrm{d} Z} \frac{1}{2 \eta(Z)} \cdot \frac{R_{0}(Z)}{R_{0}(Z)^{2}-R_{1}(Z)^{2}} \cdot\left(\frac{r^{2}}{R_{0}(Z)-R_{1}(Z)}-\frac{R_{0}(Z) \cdot(1+2 \log r)}{2}\right) \\
& +\varepsilon\left(\frac{\mathrm{d}}{\mathrm{d} Z} \frac{1}{\eta(Z)}\right) \cdot \frac{R_{0}(Z) \cdot R_{1}(Z)}{4\left(R_{0}(Z)-R_{1}(Z)\right)} \cdot(1+2 \log r)+O\left(\varepsilon^{3}\right) .
\end{aligned}
$$

As argued in Sect. 1, the leading AMSA order for collapsing is governed by the radial flow component $\overline{\bar{v}}_{r 0}(r, Z)$ only; meanwhile, the axial flow one $\overline{\bar{v}}_{z 1}(r, Z)$ appears as the first-order effect beyond the leading order. Restricting to the first-order AMSA, $\overline{\bar{v}}_{z 1}(r, Z)$ is determined, according to (3.26), as a linear function of $\mathrm{d}(1 / \eta(Z)) / \mathrm{d} Z, \mathrm{~d} R_{0}(Z) / \mathrm{d} Z$, and $\mathrm{d} R_{1}(Z) / \mathrm{d} Z$. The courses of the latter functions even characterize the slow axial parameter dependence discussed in Sects. 3.1 and 3.2.

We subdivide $\overline{\bar{v}}_{z 1}(r, Z)$ into a kinematic part ${ }^{(\text {kin })} \overline{\bar{v}}_{z 1}^{(0)} \cdot \mathrm{d} R_{0}(Z) / \mathrm{d} Z+{ }^{(\text {kin })} \overline{\bar{v}}_{z 1}^{(1)} \cdot \mathrm{d} R_{1}(Z) / \mathrm{d} Z$, and a non-kinematic one ${ }^{(\eta)} \overline{\bar{v}}_{z 1} \cdot \mathrm{d}(1 / \eta(Z)) / \mathrm{d} Z$. In the lowest order, the former quantifies that part of the axial flow component generated through the axial change of the boundary profiles of the collapsing tube, meanwhile the latter describes the axial flow contribution due to the axial change of the viscosity only. As shown in Appendix 3, both the contributions arise from the tangential force balance at the boundaries, taken into account within the first-order AMSA. We write

$\overline{\bar{v}}_{z 1}={ }^{(\operatorname{kin})} \overline{\bar{v}}_{z 1}^{(0)}(r, Z) \cdot \mathrm{d} R_{0}(Z) / \mathrm{d} Z+{ }^{(\operatorname{kin})} \overline{\bar{v}}_{z 1}^{(1)}(r, Z) \cdot \mathrm{d} R_{1}(Z) / \mathrm{d} Z+{ }^{(\eta)} \overline{\bar{v}}_{z 1}(r, Z) \cdot \mathrm{d}(1 / \eta(Z)) / \mathrm{d} Z$.

The radial flow as well as both the parts of the axial flow component depend upon governing parameters entering the collapsing problem in a quite distinct way. For a rough estimation, we introduce the variable wall thickness $W(Z)=R_{0}(Z)-R_{1}(Z)$ of the collapsing tube, approaching $R_{0}(Z) \approx 1, W(Z) \approx w, r \approx 1,1 / \eta(Z) \approx 1$, $|\mathrm{d}(1 / \eta(Z)) / \mathrm{d} Z| \approx \beta$. The dummy $w$ and $\beta$ may estimate the wall thickness and the axial distance where the reciprocal viscosity significantly changes, respectively. We find 


$$
\begin{aligned}
\left|\overline{\bar{v}}_{r 0}\right| & \approx 1 / 2 w, \\
\left|{ }^{(k i n)} \overline{\bar{v}}_{z 1}^{(0)} \frac{\mathrm{d} R_{0}}{\mathrm{~d} Z}+{ }^{(\text {kin })} \overline{\bar{v}}_{z 1}^{(1)} \frac{\mathrm{d} R_{1}}{\mathrm{~d} Z}\right| & \approx \frac{\mathrm{d} R_{0}}{\mathrm{~d} Z} \cdot \frac{1}{2 w^{2}}, \\
\left|{ }^{(\eta)} \overline{\bar{v}}_{z 1} \cdot \mathrm{d}(1 / \eta(Z)) / \mathrm{d} Z\right| & \approx \beta / 4 w .
\end{aligned}
$$

\subsection{Kinematics}

\subsubsection{Kinematic equations in AMSA}

We discuss the kinematic equations in comoving coordinates (2.16) and dimensionless units of Sect. 3.1. Because AMSA takes into account a successive boundary distortion as long as the boundary inclination is "slow," the r.h.s. of (2.16) must be of the order $\tilde{h} / \tilde{L}$. Therefore, the dimensionless heat source velocity $u$ is subject to restrictions in AMSA. As shown above, the viscous flow derived beyond the leading order of AMSA depends upon the first (and higher)-order derivatives of $R_{0}(Z)$ and $R_{1}(Z)$, respectively. Therefore, the kinematic equations (2.16) become implicit differential equations. The transformation in explicit form requires additional restrictions.

We start with expanding the r.h.s. of (2.16) in powers of $\varepsilon$, based on (3.23), (3.24). The explicit kinematic equations in the first order in $\varepsilon$ and the boundary conditions read

$$
\begin{aligned}
\mathrm{d} R_{0} / \mathrm{d} Z= & -\overline{\bar{v}}_{r 0}\left(R_{0}, Z\right) / u+\varepsilon \cdot \overline{\bar{v}}_{r 0}\left(R_{0}, Z\right) \cdot(\eta) \overline{\bar{v}}_{z 1}\left(R_{0}, Z\right) \cdot[\mathrm{d}(1 / \eta(Z)) / \mathrm{d} Z] / u^{2} \\
& -\varepsilon \cdot \overline{\bar{v}}_{r 0}\left(R_{0}, Z\right)^{2} \cdot(\operatorname{kin}) \overline{\bar{v}}_{z 1}^{(0)}\left(R_{0}, Z\right) / u^{3} \\
& -\varepsilon \cdot \overline{\bar{v}}_{r 0}\left(R_{0}, Z\right) \cdot \overline{\bar{v}}_{r 0}\left(R_{1}, Z\right) \cdot{ }^{(k i n)} \overline{\bar{v}}_{z 1}^{(1)}\left(R_{0}, Z\right) / u^{3}+O\left(\varepsilon^{2}\right), \\
\mathrm{d} R_{1} / \mathrm{d} Z= & -\overline{\bar{v}}_{r 0}\left(R_{1}, Z\right) / u+\varepsilon \cdot \overline{\bar{v}}_{r 0}\left(R_{1}, Z\right) \cdot{ }^{(\eta)} \overline{\bar{v}}_{z 1}\left(R_{1}, Z\right) \cdot[\mathrm{d}(1 / \eta(Z)) / \mathrm{d} Z] / u^{2} \\
& -\varepsilon \cdot \overline{\bar{v}}_{r 0}\left(R_{1}, Z\right)^{2} \cdot \cdot^{(\mathrm{kin})} \overline{\bar{v}}_{z 1}^{(0)}\left(R_{1}, Z\right) / u^{3} \\
& -\varepsilon \cdot \overline{\bar{v}}_{r 0}\left(R_{1}, Z\right) \cdot \overline{\bar{v}}_{r 0}\left(R_{0}, Z\right) \cdot{ }^{(\mathrm{kin})} \overline{\bar{v}}_{z 1}^{(1)}\left(R_{1}, Z\right) / u^{3}+O\left(\varepsilon^{2}\right), \\
\lim _{Z \rightarrow \infty} R_{0}(Z)= & 1, \lim _{Z \rightarrow \infty} R_{1}(Z)=R_{1 \max }<1 .
\end{aligned}
$$

The "small" boundary inclination is governed through $-\overline{\bar{v}}_{r 0} / u$. This restricts $u$ according to $\left|\mathrm{d} R_{0} / \mathrm{d} Z\right| \approx$ $\left|\mathrm{d} R_{1} / \mathrm{d} Z\right| \approx 1 / 2 w u \ll 1$, as seen using (3.28). The additional perturbation treatment to derive the explicit kinematic equations (3.31), (3.32) requires that the kinematic axial flow (3.29) must not overcome the radial flow (3.28). Thus, we arrive at a stronger restriction for $u$ according to

$1 / 2 w^{2} u \ll 1$

\subsubsection{Wall thickness and boundary profiles in the zeroth order (1D theory)}

Restricting to the zeroth order in $\varepsilon$ on the r.h.s. of (3.31), (3.32), we obtain the kinematic equations of the 1D theory:

$\mathrm{d} R_{00}(Z) / \mathrm{d} Z=(1 / u) \cdot(1 / 2 \eta(Z)) \cdot\left(R_{10}(Z) /\left(R_{00}(Z)-R_{10}(Z)\right)\right)$,

$\mathrm{d} R_{10}(Z) / \mathrm{d} Z=(1 / u) \cdot(1 / 2 \eta(Z)) \cdot\left(R_{00}(Z) /\left(R_{00}(Z)-R_{10}(Z)\right)\right)$,

where $R_{00}(Z)$ and $R_{10}(Z)$ denote the course of the outer and inner boundaries, respectively, in the zeroth order of $\varepsilon$. (3.35), (3.36) together with (3.33) can be exactly solved. We introduce $W(\infty)=1-R_{1 \max }, \Delta W(Z)=$ $W(Z)-W(\infty)$, and $\Delta W^{\text {tot }}=\Delta W(-\infty)$ as wall thickness at start, change of the wall thickness from start, and total change of the wall thickness after collapsing, respectively. The change of the wall thickness $\Delta W_{0}(Z)$, and the wall thickness $W_{0}(Z)$ in zeroth order (1D theory) are directly determined by subtracting (3.36) from (3.35) according to 


$$
\begin{aligned}
\Delta W_{0}(Z) & =(1 / 2 u) \cdot \int_{Z}^{\infty} \mathrm{d} Z_{1} \cdot\left(1 / \eta\left(Z_{1}\right)\right), \\
W_{0}(Z) & =1-R_{1 \max }+\Delta W_{0}(Z),
\end{aligned}
$$

respectively. The total change of the wall thickness in the zeroth order is

$$
\Delta W_{0}^{(\mathrm{tot})}=(1 / 2 u) \cdot \int_{-\infty}^{\infty} \mathrm{d} Z(1 / \eta(Z))=\Delta Z_{H} / 2 u
$$

(see also [3] for an intuitive 1D analysis). The characteristic dimensionless axial distance

$\Delta Z_{H}=\int_{-\infty}^{\infty} \mathrm{d} Z(1 / \eta(Z))$

may be taken as a measure of the axial length of both the heating zone and the collapsing cone. Multiplying (3.35) and (3.36) with $R_{00}(Z)$ and $R_{10}(Z)$, respectively, and subtracting, we get

$$
R_{00}(Z)^{2}-R_{10}(Z)^{2}=1-R_{1 \max }^{2}=\text { const },-\infty \leq Z \leq \infty .
$$

Equation (3.41) is the principle of local mass conservation within the 1D theory. Because the latter deals with an incompressible radial flow only, any local volume segment in the laboratory system with the axial length $\mathrm{d} z$ and the volume $\pi\left(R_{0}^{2}-R_{1}^{2}\right) \mathrm{d} z$ must keep its volume constant during collapsing. Combining (3.38), (3.39), and (3.41), we find

$R_{00}(Z)=(1 / 2) \cdot\left(1-R_{1 \max }^{2}+W_{0}(Z)^{2}\right) / W_{0}(Z)$,

and $R_{10}(Z)=R_{00}(Z)-W_{0}(Z)$. Independently of any hydrodynamic approaches, the principle of local mass conservation always holds true beyond the collapsing zone where the boundary inclination can be neglected, and the total viscous flow is suppressed. Then (3.42) is generalized to

$R_{0 \min }=\frac{1}{2} \cdot \frac{1-R_{1 \max }^{2}+\left(1-R_{1 \max }+\Delta W^{(\mathrm{tot})}\right)^{2}}{1-R_{1 \max }+\Delta W^{(\mathrm{tot})}}$.

The 1D formulae of collapsing-relevant geometry parameters are substantially simplified if the reciprocal viscosity in comoving coordinates and dimensionless units is given as Gaussian according to

$1 / \eta(Z)=\exp \left(-\alpha^{2} Z^{2}\right)$

As a measure of the variation of $1 / \eta(Z), \alpha$ replaces the general parameter $\beta$ introduced to estimate (3.27). Then (3.37) and (3.40) become specified to

$$
\begin{aligned}
\Delta W_{0}(Z) & =(\sqrt{\pi} / 4 \alpha u) \cdot(1-\operatorname{erf}(\alpha Z)), \\
\Delta Z_{H} & =\sqrt{\pi} / \alpha .
\end{aligned}
$$

In physical units, we have $\alpha=R_{0 \max } / z_{e}$, with $z= \pm z_{e}$ as the positions where the viscosity reaches its $e$-fold minimum value, so that $2 z_{e}$ is a measure of the axial width of the heating zone. $\alpha$ depends upon the tube radii and details of the heat sources chosen in practice. Rather small $\alpha$, e.g., $\alpha \leq 0.5$, may be expected, e.g., for cylindrical furnaces surrounding the glass tube (see, e.g., [15]). On the other hand, a rather small heating zone and a large $\alpha$ value up to 2.5 can be reached for circularly arranged torches around the tube $[3,4]$. 


\subsubsection{Axial flow and first-order corrections (2D theory)}

Extending the 1D collapsing kinematics owing to the presence of the axial viscous flow, we will turn from the 1D to the 2D theory, as argued in Sect. 1. We will distinguish kinematic and non-kinematic 2D corrections designated by (kin) and $(\eta)$, respectively, according to (3.27). The kinematic and non-kinematic parts of the axial flow, related to the governing radial flow of the 1D theory, are of the orders $1 /\left(u \cdot w^{2}\right)$ and $\beta$, respectively, as seen from (3.27) to (3.30) and (3.34). The non-kinematic part of the axial flow dominates the kinematic one for $u \gg\left(1 /\left(\beta \cdot w^{2}\right)\right.$. Making use of $1 / \beta \approx \Delta Z_{H}$ if $1 / \eta(Z)$ is given as an exponential introduced in Sect. 3.3, the former condition can be rewritten as $\Delta Z_{H} / u \ll w^{2}<1-R_{1 \max }$. This means that, according to (3.38), (3.39), the non-kinematic axial flow will dominate the kinematic one if the total change of the wall thickness is sufficiently smaller than the starting wall thickness, which is the case for sufficiently large $u$.

The 2D theory results in $u$-dependent corrections to the 1D approaches of collapsing-relevant geometry parameters. For calculation, we introduce the perturbation expansions of the outer tube radius and wall thickness according to

$$
\begin{aligned}
& R_{0}(Z)=R_{00}(Z)+\varepsilon R_{01}(Z)+\cdots, \\
& W(Z)=W_{0}(Z)+\varepsilon W_{1}(Z)+\cdots,
\end{aligned}
$$

where $R_{00}(Z), W_{0}(Z)$ are known from (3.42), (3.38), respectively. Remarkably, the 2D corrections for $R_{0 \mathrm{~min}}$ and $R_{1 \text { min }}$ are exceptionally small, provided that the preconditions of AMSA hold true. We only outline the basic results and estimations and refer to Appendix 4 for some details.

As shown in Appendix 4, the first-order correction $\Delta W_{1}^{(\text {tot })}=W_{1}(-\infty)-W_{1}(\infty)$ to the total change $\Delta W^{(\text {tot })}$ of the wall thickness across the collapsing profile is found as a power series in $1 / u$. The latter starts with $1 / u^{2}$ and $1 / u^{3}$, due to the non-kinematic and kinematic $2 \mathrm{D}$ corrections, respectively. Remarkably, the $1 / u^{2}$ term cancels for $\mathrm{ABC}$ chosen at infinity. In subsequent order $1 / u^{3}, \Delta W_{1}^{(\mathrm{tot})}$ is governed by the kinematic axial flow. We arrive at

$$
\Delta W_{1}^{(\mathrm{tot})}=-\frac{1}{8 u^{3}\left(1-R_{1 \max }\right)^{3}} \int_{-\infty}^{\infty} \mathrm{d} Z \cdot\left(1 / \eta(Z)^{3}\right) \cdot\left(1+O(w)+O\left(\Delta Z_{H} / w u\right)\right) .
$$

If $1 / \eta(Z)$ is specified as Gaussian (3.44), the integral in (3.49) equals $\Delta Z_{H} / \sqrt{3}$.

The $2 \mathrm{D}$ correction to the outer radius $R_{0 \mathrm{~min}}$ after collapsing is calculated according to $\lim _{Z \rightarrow-\infty} R_{01}(Z)$. If (3.49) holds true, we have from (3.43) for large $u$

$\lim _{Z \rightarrow-\infty} R_{01}(Z)=\Delta W_{1}^{(\text {tot })} /\left(1-R_{1 \max }\right)$,

the order of which is $\lim _{Z \rightarrow-\infty} R_{01}(Z) \approx \Delta Z_{H} / u^{3} w^{4}$. We will compare (3.50) with the leading part in $1 / u$ of the $1 \mathrm{D}$ result. The $1 \mathrm{D}$ approach of $R_{0 \mathrm{~min}}$ in the large $u$ limit, derived from (5.32), is

$R_{00 \min }=1-\frac{\Delta Z_{H}}{2 u} \frac{R_{1 \max }}{1-R_{1 \max }}+O\left(1 / w^{2} u^{2}\right)$.

The 2D correction (3.50) is small of the order $\left.w \cdot\left(1 / u w^{2}\right)\right)^{2}$, compared with the second term on the r.h.s. of (3.51) and, therefore, is negligible, regarding the general AMSA condition (3.34).

The low order of magnitude and strong parameter dependence of the 2D correction (3.50) are understood, on the one hand, through the wide cancelation of the non-kinematic axial flow contribution and, on the other hand, through the double role of the boundary inclination in AMSA, being kinematic and perturbing effect as well.

\subsubsection{On the second-order and higher-order $2 D$ corrections}

The AMSA established in this work leads to perturbation series of the viscous flow proceeding in $\varepsilon^{2}$. We, therefore, expect that if the preconditions of AMSA hold true, higher AMSA perturbation orders will result in a minor 
numerical refinement only, compared with the 1D theory and the first-order 2D corrections. For completeness, we outline the second-order total change of the wall thickness $\Delta W_{2}^{\text {(tot) }}$, calculated from the second-order normal force balance. We find in the large $u$ limit

$\Delta W_{2}^{(\text {tot })}=-\frac{5+7 \cdot\left(1-R_{1 \max }\right)^{2}}{64 u^{3}\left(1-R_{1 \max }\right)^{2}} \cdot \int_{-\infty}^{\infty} \mathrm{d} Z \frac{1}{\eta(Z)^{3}} \cdot\left(1+O(w)+O\left(\Delta Z_{H} / u w\right)\right)$,

which is smaller by a factor of about $w$ compared to the first-order result (3.49).

For completeness, too, conditions are of interest where leading $2 \mathrm{D}$ contributions to $\Delta W^{(\text {tot })}$ come from the nonkinematic perturbation only, remembering that the axial viscosity dependence has been introduced in AMSA as the fundamental non-kinematic perturbation. The analysis can be enforced by suppressing the boundary inclination through the ad hoc restriction $R_{0}(Z)=R_{0 \max }=$ const, $R_{1}(Z)=R_{1 \max }=$ const. With this information, we extended AMSA up to $\varepsilon^{4}$, where the analytic effort remains controllable with the aid of computer-supported tools. We will restrict here to point out that up to the fourth AMSA perturbation order, the non-kinematic contributions $\propto 1 / u^{2}$ to $\Delta W^{\text {(tot) }}$ cancel for arbitrary viscosity courses given as exponential according to Sect. 3.3.

The result from all parts of the outlined perturbation analysis, valid for $1 / u w \ll 1, \beta w \ll 1$, may be roughly summarized as

$\Delta W^{(\text {tot })}=\left(\Delta Z_{H} / 2 u\right) \cdot\left(1+O\left(1 / u^{2} w^{3}\right)+O\left(\beta^{5} / u w\right)+O\left(\beta^{6}\right)\right)$.

The first remainder in (3.53) stands for the kinematic 2D correction of the 1D result, including the second-order effects, whereas the second and third remainders estimate non-kinematic corrections, based on the correct results of the AMSA perturbation analysis in $\varepsilon^{4}$.

\section{Discussion}

\subsection{General remarks}

Free boundaries and a variable viscosity characterize collapsing as an outstanding hydrodynamic problem beyond common analytic tools of mathematical physics. Analytic approaches only facilitate the detailed physical understanding and knowledge of basic parameter dependencies of this complex phenomenon. If collapsing is applied to measure the temperature-dependent glass viscosity [3,4], correct analytic approaches of geometry parameters can significantly reduce the effort of data evaluation, compared with finite element (FEM) simulations. On the other hand, FEM simulations can verify the consistency of analytic approaches, and vice versa, correct analytic approaches allow for a rigorous test of FEM algorithms. The latter aspect is important insofar as the same FEM algorithms will be extended, of course, to parameter areas where analytical approaches fail.

In the following, the dimensionless results from above will be rewritten in physical (dimensional) quantities. We will focus on the "shrinking ratio" (outer radius after collapsing $R_{0 \min }$ divided by the outer radius $R_{0 \max }$ before collapsing), as it depends upon several parameters entering the collapsing problem. The reciprocal viscosity in comoving coordinates may be given as Gaussian (3.44). Comparative FEM calculations have been carried out with the $\operatorname{COMSOL}^{(R)}$ Multiphysics $4.2 \mathrm{a}$ program package in laboratory coordinates and physical units, to simulate collapsing as an advancing time-dependent process, like in laboratory. A rigid wall cut-off of the viscosity course at $\eta(z)=10^{12} \mathrm{~Pa}$ s, corresponding to $10^{7} \eta_{\mathrm{min}}$, has been introduced, and the successive shape deformation across the collapsing cone has been taken into account through the ALE moving mesh module in COMSOL. $R_{0 \min } / R_{0 \max }$ has been determined with an accuracy of $10^{-4}$.

In Fig. 1a, b, we show the fitted results of $R_{0 \min } / R_{0 \max }$ from FEM. The parameters are $R_{1 \max } / R_{0 \max }=0.7$ and $0.9,0.20 \leq \alpha \leq 1.00$, where $\alpha=R_{0 \max } / z_{e}$. 
a

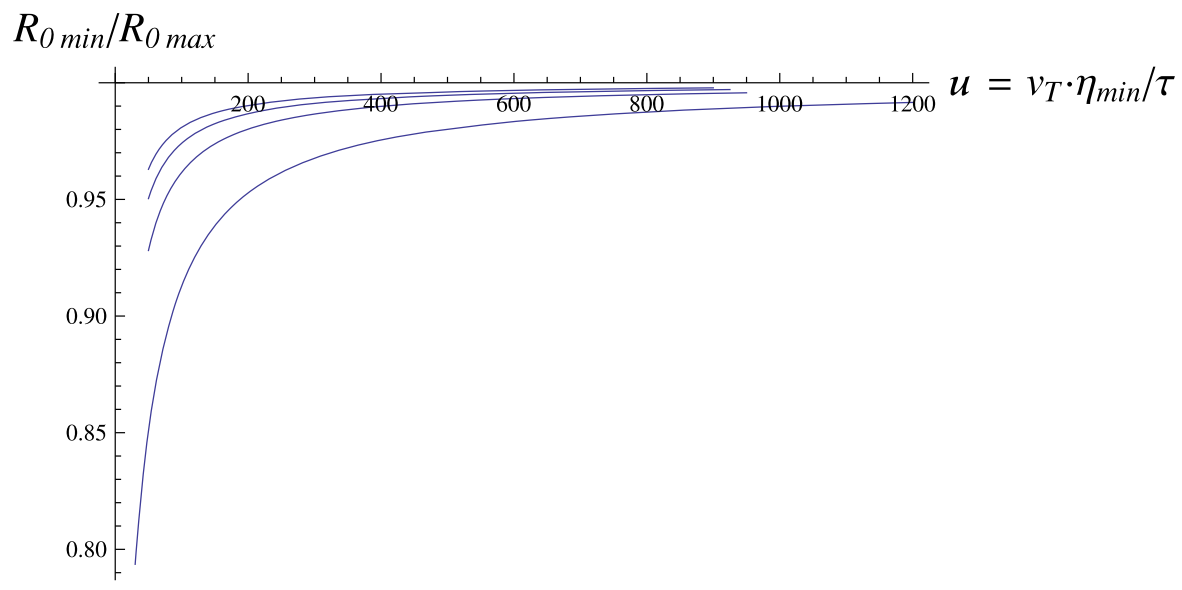

b

$R_{0 \text { min }} / R_{0 \text { max }}$

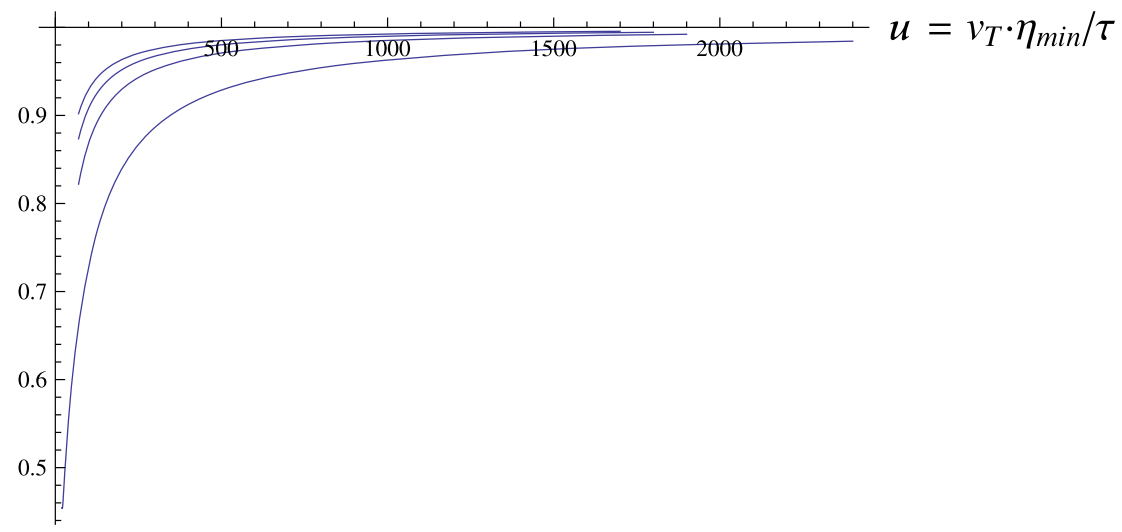

Fig. 1 Shrinking ratio $R_{0 \min } / R_{0 \max }$ versus dimensionless heat source velocity $u=v_{T} \cdot \eta_{\min } / \tau$ (both rewritten in physical units) from fits of FEM simulations for the Gaussian course (3.44) of the reciprocal viscosity. Parameter: $\alpha=R_{0 \text { max }} / z_{e}$ (rewritten in physical units). a $R_{1 \max } / R_{0 \max }=0.7, \alpha=0.20,0.50,0.75,1.00$ (from below); b $R_{1 \max } / R_{0 \max }=0.9, \alpha=0.20,0.50,0.75,1.00$ (from below)

Table $1 R_{1 \max } / R_{0 \max }=0.7, u=50$

\begin{tabular}{lllll}
\hline$\alpha$ & $1-R_{0 \min } / R_{0 \max }$ & $\begin{array}{l}1-R_{0 \min } / R_{0 \max } \\
1 \mathrm{D} \text { theory }\end{array}$ & $\begin{array}{l}1-R_{0 \min } / R_{0 \max } \\
\text { 2D theory }\end{array}$ & $\begin{array}{l}\text { Estim. abs. error } \\
\text { Column 3 and 4 }\end{array}$ \\
\hline 0.125 & $0.2019 \pm 0.0001$ & 0.20191 & 0.20184 & \pm 0.002 \\
0.20 & $0.1494 \pm 0.0001$ & 0.14952 & 0.14941 & \pm 0.002 \\
0.50 & $0.0719 \pm 0.0001$ & 0.07210 & 0.07204 & \pm 0.002 \\
0.75 & $0.0496 \pm 0.0001$ & 0.05025 & 0.05020 & \pm 0.01 \\
1.00 & $0.0371 \pm 0.0001$ & 0.03855 & 0.03851 & \pm 0.04 \\
\end{tabular}

Comparison of $1-R_{0 \min } / R_{0 \max }$ for Gaussian course (3.44) of the reciprocal viscosity and decreasing axial length of the collapsing zone. 2nd column: from discrete FEM simulation. 3rd column: 1D theory (zeroth-order AMSA, (3.43), (3.39), (3.46)). 4th column: $2 \mathrm{D}$ theory (second-order AMSA, see text). 5th column: estimated absolute error from AMSA (see text). Dimensionless parameters in physical units: $u=v_{T} \cdot \eta_{\min } / \tau, \alpha=R_{0 \max } / z_{e}$ 
Table $2 R_{1 \max } / R_{0 \max }=0.7, u=200$

\begin{tabular}{lllll}
\hline$\alpha$ & $\begin{array}{l}1-R_{0 \min } / R_{0 \max } \\
\text { FEM }\end{array}$ & $\begin{array}{l}1-R_{0 \min } / R_{0 \max } \\
1 \mathrm{D} \text { theory }\end{array}$ & $\begin{array}{l}1-R_{0 \min } / R_{0 \max } \\
2 \mathrm{D} \text { theory }\end{array}$ & $\begin{array}{l}\text { Estim. abs. error } \\
\text { Columns 3 and } 4\end{array}$ \\
\hline 0.125 & $0.0720 \pm 0.0001$ & 0.07210 & 0.07210 & $\pm 4 \times 10^{-5}$ \\
0.20 & $0.0472 \pm 0.0001$ & 0.04738 & 0.04738 & $\pm 2 \times 10^{-5}$ \\
0.50 & $0.0199 \pm 0.0001$ & 0.01995 & 0.01996 & \pm 0.0003 \\
0.75 & $0.0132 \pm 0.0001$ & 0.01346 & 0.01346 & \pm 0.002 \\
1.00 & $0.0097 \pm 0.0001$ & 0.01015 & 0.01015 & \pm 0.01
\end{tabular}

Comparison of $1-R_{0 \min } / R_{0 \max }$ for Gaussian course (3.44) of the reciprocal viscosity and decreasing axial length of the collapsing zone. 2nd column: from discrete FEM simulation. 3rd column: 1D theory (zeroth-order AMSA, (3.43), (3.39), (3.46)). 4th column: 2D theory (second-order AMSA, see text). 5th column: estimated absolute error from AMSA (see text). Dimensionless parameters in physical units: $u=v_{T} \cdot \eta_{\min } / \tau, \alpha=R_{0 \max } / z_{e}$

Table $3 R_{1 \max } / R_{0 \max }=0.9, u=50$

\begin{tabular}{lllll}
\hline$\alpha$ & $\begin{array}{l}1-R_{0 \min } / R_{0 \max } \\
\text { FEM }\end{array}$ & $\begin{array}{l}1-R_{0 \min } / R_{0 \max } \\
1 \mathrm{D} \text { theory }\end{array}$ & $\begin{array}{l}1-R_{0 \min } / R_{0 \max } \\
2 \mathrm{D} \text { theory }\end{array}$ & $\begin{array}{l}\text { Estim. abs. error } \\
\text { Columns } 3 \text { and } 4\end{array}$ \\
\hline 0.125 & $0.4867 \pm 0.0001$ & 0.48621 & 0.48569 & \pm 0.005 \\
0.20 & $0.4029 \pm 0.0001$ & 0.40204 & 0.40098 & \pm 0.004 \\
0.50 & $0.2316 \pm 0.0001$ & 0.23090 & 0.22913 & \pm 0.007 \\
0.75 & $0.1683 \pm 0.0001$ & 0.16978 & 0.16805 & \pm 0.04 \\
1.00 & $0.1320 \pm 0.0001$ & 0.13417 & 0.13258 & \pm 0.16
\end{tabular}

Comparison of $1-R_{0 \min } / R_{0 \max }$ for Gaussian course (3.44) of the reciprocal viscosity and decreasing axial length of the collapsing zone. 2nd column: from discrete FEM simulation. 3rd column: 1D theory (zeroth-order AMSA, (3.43), (3.39), (3.46)). 4th column: 2D theory (second-order AMSA, see text). 5th column: estimated absolute error from AMSA (see text). Dimensionless parameters in physical units: $u=v_{T} \cdot \eta_{\min } / \tau, \alpha=R_{0 \max } / z_{e}$

Table $4 R_{1 \max } / R_{0 \max }=0.9, u=200$

\begin{tabular}{lllll}
\hline$\alpha$ & $\begin{array}{l}1-R_{0 \min } / R_{0 \max } \\
\text { FEM }\end{array}$ & $\begin{array}{l}1-R_{0 \min } / R_{0 \max } \\
1 \mathrm{D} \text { theory }\end{array}$ & $\begin{array}{l}1-R_{0 \min } / R_{0 \max } \\
2 \mathrm{D} \text { theory }\end{array}$ & $\begin{array}{l}\text { Estim. abs. error } \\
\text { Columns 3 and 4 }\end{array}$ \\
\hline 0.125 & $0.2308 \pm 0.0001$ & 0.23090 & 0.23079 & \pm 0.0001 \\
0.20 & $0.1607 \pm 0.0001$ & 0.16122 & 0.16112 & \pm 0.0001 \\
0.50 & $0.0703 \pm 0.0001$ & 0.07290 & 0.07284 & \pm 0.001 \\
0.75 & $0.0482 \pm 0.0001$ & 0.05004 & 0.04999 & \pm 0.009 \\
1.00 & $0.0366 \pm 0.0001$ & 0.03809 & 0.03805 & \pm 0.04
\end{tabular}

Comparison of $1-R_{0 \min } / R_{0 \max }$ for Gaussian course (3.44) of the reciprocal viscosity and decreasing axial length of the collapsing zone. 2nd column: from discrete FEM simulation. 3rd column: 1D theory (zeroth-order AMSA, (3.43), (3.39), (3.46)). 4th column: 2D theory (second-order AMSA, see text). 5th column: estimated absolute error from AMSA (see text). Dimensionless parameters in physical units: $u=v_{T} \cdot \eta_{\min } / \tau, \alpha=R_{0 \max } / z_{e}$

\subsection{AMSA compared with FEM}

Numerical data of $1-R_{0 \min } / R_{0 \max }$ from FEM, from the analytic 1D and 2D theories, and the estimated absolute error of the AMSA data are outlined in Tables 1-8. The 1D data have been calculated from the zeroth-order AMSA formulae, combining (3.43), (3.39), and (3.46). The 2D data are based, in addition, on the AMSA formulae up to the second order (5.29) to (5.31), (5.35), and (3.52). The fifth column of Tables 1-8 outlines the estimations of absolute uncertainties of AMSA data via extrapolation to non-performed higher-order perturbation steps. They 
Table $5 R_{1 \max } / R_{0 \max }=0.7, \alpha=0.125$

\begin{tabular}{lllll}
\hline$u$ & $\begin{array}{l}1-R_{0 \min } / R_{0 \max } \\
\text { FEM }\end{array}$ & $\begin{array}{l}1-R_{0 \min } / R_{0 \max } \\
1 \mathrm{D} \text { theory }\end{array}$ & $\begin{array}{l}1-R_{0 \min } / R_{0 \max } \\
2 \mathrm{D} \text { theory }\end{array}$ & $\begin{array}{l}\text { Estim. abs. error } \\
\text { Columns 3 and } 4\end{array}$ \\
\hline 50 & $0.2019 \pm 0.0001$ & 0.20191 & 0.20184 & \pm 0.002 \\
100 & $0.1269 \pm 0.0001$ & 0.12703 & 0.12701 & \pm 0.0003 \\
200 & $0.0720 \pm 0.0001$ & 0.07210 & 0.07209 & $\pm 4 \times 10^{-5}$ \\
500 & $0.0312 \pm 0.0001$ & 0.03127 & 0.03127 & $\pm 2 \times 10^{-6}$ \\
1000 & $0.0160 \pm 0.0001$ & 0.01608 & 0.01608 & $\pm 2 \times 10^{-7}$
\end{tabular}

Comparison of $1-R_{0 \min } / R_{0 \max }$ for Gaussian course (3.44) of the reciprocal viscosity and decreasing axial length of the collapsing zone. 2nd column: from discrete FEM simulation. 3rd column: 1D theory (zeroth-order AMSA, (3.43), (3.39), (3.46)). 4th column: 2D theory (second-order AMSA, see text). 5th column: estimated absolute error from AMSA (see text). Dimensionless parameters in physical units: $u=v_{T} \cdot \eta_{\min } / \tau, \alpha=R_{0 \max } / z_{e}$

Table $6 R_{1 \max } / R_{0 \max }=0.7, \alpha=0.50$

\begin{tabular}{lllll}
\hline$u$ & $1-R_{0 \min } / R_{0 \max }$ & $\begin{array}{l}1-R_{0 \min } / R_{0 \max } \\
\text { FEM theory }\end{array}$ & $\begin{array}{l}1-R_{0 \min } / R_{0 \max } \\
\text { 2D theory }\end{array}$ & $\begin{array}{l}\text { Estim. abs. error } \\
\text { Columns 3 and 4 }\end{array}$ \\
\hline 4.58 & $0.2850 \pm 0.0001$ & 0.28532 & 0.28475 & \pm 0.2 \\
12.7 & $0.2002 \pm 0.0001$ & 0.20010 & 0.19897 & \pm 0.04 \\
30 & $0.1100 \pm 0.0001$ & 0.11031 & 0.11009 & \pm 0.006 \\
100 & $0.0384 \pm 0.0001$ & 0.03855 & 0.03854 & \pm 0.0008 \\
500 & $0.0081 \pm 0.0001$ & 0.00815 & 0.00815 & \pm 0.0001
\end{tabular}

Comparison of $1-R_{0 \min } / R_{0 \max }$ for Gaussian course (3.44) of the reciprocal viscosity and decreasing axial length of the collapsing zone. 2nd column: from discrete FEM simulation. 3rd column: 1D theory (zeroth-order AMSA, (3.43), (3.39), (3.46)). 4th column: 2D theory (second-order AMSA, see text). 5th column: estimated absolute error from AMSA (see text). Dimensionless parameters in physical units: $u=v_{T} \cdot \eta_{\min } / \tau, \alpha=R_{0 \max } / z_{e}$

Table $7 R_{1 \max } / R_{0 \max }=0.9, \alpha=0.125$

\begin{tabular}{lllll}
\hline$u$ & $\begin{array}{l}1-R_{0 \min } / R_{0 \max } \\
\text { FEM }\end{array}$ & $\begin{array}{l}1-R_{0 \min } / R_{0 \max } \\
1 \mathrm{D} \text { theory }\end{array}$ & $\begin{array}{l}1-R_{0 \min } / R_{0 \max } \\
2 \mathrm{D} \text { theory }\end{array}$ & $\begin{array}{l}\text { Estim. abs. error } \\
\text { Columns 3 and } 4\end{array}$ \\
\hline 50 & $0.4867 \pm 0.0001$ & 0.48621 & 0.48569 & \pm 0.005 \\
100 & $0.3591 \pm 0.0001$ & 0.35866 & 0.35833 & \pm 0.0009 \\
200 & $0.2309 \pm 0.0001$ & 0.23090 & 0.23079 & \pm 0.0001 \\
500 & $0.1107 \pm 0.0001$ & 0.11089 & 0.11087 & $\pm 7 \times 10^{-6}$ \\
1000 & $0.0593 \pm 0.0001$ & 0.05935 & 0.05934 & $\pm 9 \times 10^{-7}$
\end{tabular}

Comparison of $1-R_{0 \min } / R_{0 \max }$ for Gaussian course (3.44) of the reciprocal viscosity and decreasing axial length of the collapsing zone. 2nd column: from discrete FEM simulation. 3rd column: 1D theory (zeroth-order AMSA, (3.43), (3.39), (3.46)). 4th column: 2D theory (second-order AMSA, see text). 5th column: estimated absolute error from AMSA (see text). Dimensionless parameters in physical units: $u=v_{T} \cdot \eta_{\min } / \tau, \alpha=R_{0 \max } / z_{e}$

summarize non-kinematic contributions, based on the second and third remainders of (3.53) (with $\beta=\alpha$ ), and the kinematic ones from the remainders in (3.49) and (3.52). Tables 1, 2, 3 and 4 are arranged to demonstrate the limit of AMSA with respect to the non-kinematic perturbation for increasing $\alpha$, and Tables 5, 6, 7 and 8 with respect to the kinematic perturbation for decreasing $u$. In particular, the data of both Tables 6 and 8 nearly approach the geometric collapsing limit $R_{1 \min } \rightarrow 0$ that would be reached for $1-R_{0 \min } / R_{0 \max } \rightarrow 0.286$ if $R_{1 \max } / R_{0 \max }=0.7$, and $1-R_{0 \min } / R_{0 \max } \rightarrow 0.564$ if $R_{1 \max } / R_{0 \max }=0.9$. 
Table $8 R_{1 \max } / R_{0 \max }=0.9, \alpha=0.50$

\begin{tabular}{lllll}
\hline$u$ & $\begin{array}{l}1-R_{0 \min } / R_{0 \max } \\
\text { FEM }\end{array}$ & $\begin{array}{l}1-R_{0 \min } / R_{0 \max } \\
1 \mathrm{D} \text { theory }\end{array}$ & $\begin{array}{l}1-R_{0 \min } / R_{0 \max } \\
\text { 2D theory }\end{array}$ & $\begin{array}{l}\text { Estim. abs. error } \\
\text { Column 3 and 4 }\end{array}$ \\
\hline 10 & $0.5219 \pm 0.0001$ & 0.51872 & 0.51041 & \pm 0.2 \\
20 & $0.4039 \pm 0.0001$ & 0.40204 & 0.39535 & \pm 0.04 \\
50 & $0.2312 \pm 0.0001$ & 0.23090 & 0.22913 & \pm 0.007 \\
200 & $0.0729 \pm 0.0001$ & 0.07290 & 0.07284 & \pm 0.001 \\
500 & $0.0307 \pm 0.0001$ & 0.03075 & 0.03074 & \pm 0.0005
\end{tabular}

Comparison of $1-R_{0 \min } / R_{0 \max }$ for Gaussian course (3.44) of the reciprocal viscosity and decreasing axial length of the collapsing zone. 2nd column: from discrete FEM simulation. 3rd column: 1D theory (zeroth-order AMSA, (3.43), (3.39), (3.46)). 4th column: 2D theory (second-order AMSA, see text). 5th column: estimated absolute error from AMSA (see text). Dimensionless parameters in physical units: $u=v_{T} \cdot \eta_{\min } / \tau, \alpha=R_{0 \max } / z_{e}$

We refer to the striking fact that, the error estimation of the AMSA data notwithstanding, the numerical differences of $1-R_{0 \min } / R_{0 \max }$ are the marginal ones, comparing the $1 \mathrm{D}$ and $2 \mathrm{D}$ results. In addition, these findings also hold true in comparison with the outlined FEM data. At least for the Gaussian (3.44), the analytic result for $1-R_{0 \min } / R_{0 \max }$ derived from the 1D theory through combining (3.43), (3.39), and (3.46) represents an excellent fit of the FEM data for the full parameter area outlined in Tables $1-8$.

Comparing the $1 \mathrm{D}$ and $2 \mathrm{D}$ theories, the minor differences of $1-R_{0 \min } / R_{0 \max }$ may be partially explained through peculiarities of the low-order AMSA perturbation analysis discussed in Sect. 3.5.3, namely the suppression of the non-kinematic perturbation. Regarding the estimated error outlined in column 5 of Tables $1-8$, the predicted accuracy of AMSA widely varies, namely with respect to $\alpha$, from being very precise beyond FEM for $\alpha \leq 0.2$ to extremely erroneous for $\alpha>0.6$. The latter region is characterized by a strong non-kinematic perturbation, so that AMSA is no longer justified from its fundamentals.

\section{Summary}

We succeed in a consistent description and understanding of collapsing of glass tubes with moving heat sources, based on a 2D axial symmetric analysis of hydrodynamic quantities established in this work. We treat the glass as an incompressible Stokes fluid and assume an axially varying viscosity. For collapsing zones exceeding the starting tube diameter, and for sufficiently large heat source velocities, but for arbitrary viscosity courses, we outline the analytic expressions of collapsing-relevant geometry parameters, which may speed up the data evaluation using collapsing as the measuring principle for the temperature-dependent glass viscosity.

We show that the asymptotic multiscale analysis (AMSA) is appropriate for a joint, consistent treatment of the Stokes equations, boundary values, and collapsing kinematics, as a prerequisite at all to arrive at analytic formulae. This advantage is gained by the concession that AMSA cannot cover the full parameter range being of interest in practice. We characterize and justify the known 1D theory as the zeroth-order AMSA, where the higher-order approaches naturally result in 2D theories.

For axial courses of the reciprocal viscosity in comoving coordinates specified as Gaussian, numerical data of the shrinkage of the external tube radius after collapsing from FEM and from the zeroth- and higher-order AMSA (1D and 2D theories) are outlined, together with the estimated error. The chosen parameter area involves and somewhat exceeds the preconditions of AMSA. We find (i) a marginal difference between the 1D and 2D theories only. These non-trivial findings are explained by the almost complete cancelation of the axial viscous flow contribution in the course of collapsing. (ii) Beyond the preconditions of AMSA, the results from AMSA agree better with FEM results than those predicted by the AMSA error estimation. Thus, formulae derived from the 1D theory result in simple and precise fits of FEM at least for the parameter area of Tables 1-8. For arbitrary viscosity courses, we also estimate a very small error of the 1D theory, provided that the preconditions of AMSA hold true and the temperature-dependent 
glass viscosity obeys an Arrhenius law. AMSA as outlined in this work cannot explain the actual validity limits of the $1 \mathrm{D}$ theory. This problem is open for further discussions.

Acknowledgements The authors thank Dr. J. Kirchhof for critical discussions and Dipl.-Ing. H.-J. Pissler †, Dr. S. Unger, and Dr. J. Kobelke for great support in the preparation of this work.

Open Access This article is distributed under the terms of the Creative Commons Attribution 4.0 International License (http:// creativecommons.org/licenses/by/4.0/), which permits unrestricted use, distribution, and reproduction in any medium, provided you give appropriate credit to the original author(s) and the source, provide a link to the Creative Commons license, and indicate if changes were made.

\section{Appendix 1: Reformulation of Stokes equation}

We substitute (2.17), (2.18) into (2.9). For reasons of consistency, we must have

$$
(1 / \eta(z)) \cdot\left(\nabla p(r, z)-\sigma^{\prime}(r, z) \cdot \nabla \eta(z)\right)=\nabla \times\left(\hat{\mathbf{e}}_{\varphi} \cdot Q^{\prime}(r, z)\right)
$$

Assuming $Q^{\prime}(r, z)$ exists such that (5.1) holds true, the Stokes equation (2.9) can be substituted, because of $\Delta \mathbf{v}=$ $\nabla \times \Delta \mathbf{A}(r, z))=\nabla \times \Delta\left(\hat{\mathbf{e}}_{\varphi} \cdot A_{\varphi}(r, z)\right), \Delta\left(\hat{\mathbf{e}}_{\varphi} \cdot A_{\varphi}(r, z)\right)=\hat{\mathbf{e}}_{\varphi} \cdot\left(\Delta-1 / r^{2}\right) A_{\varphi}(r, z)$ (see, e.g., [42]), by

$\left(\Delta-1 / r^{2}\right) A_{\varphi}(r, z)=Q^{\prime}(r, z)$.

$\mathbf{A}(r, z)$ is called vector potential because $\mathbf{A}(r, z)$ obeys a potential equation with source term. $-Q^{\prime}(r, z)$ can be described as the azimuthal vorticity component (see, e.g., [42]). If any joint functions $Q^{\prime}(r, z), A_{\varphi}(r, z)$ satisfying (5.1), (5.2) are known, the viscous flow $\mathbf{v}(r, z)$ is found by differentiation of the vector potential only.

The desired function $Q^{\prime}(r, z)$ only exists if the compatibility condition of (5.1) holds true. We find the latter by elimination of $\nabla p(r, z)$. Multiplying (5.1) with $\eta(z)$, the $r$ - and $z$-component read

$$
\begin{aligned}
& \partial p / \partial r=-\eta \cdot \partial Q^{\prime} / \partial z+\sigma_{r z}^{\prime} \cdot \mathrm{d} \eta / \mathrm{d} z, \\
& \partial p / \partial z=\eta \cdot(1 / r) \cdot(\partial / \partial r)\left(r \cdot Q^{\prime}\right)+\sigma_{z z}^{\prime} \cdot \mathrm{d} \eta / \mathrm{d} z .
\end{aligned}
$$

We subtract the $z$ - and $r$-derivative of $\partial p / \partial r$ and $\partial p / \partial z$, respectively, from each other. We arrive at (2.21),(2.22), where

$$
Q^{\prime \prime}(r, z)=\eta(z) \cdot Q^{\prime}(r, z),
$$

and (2.21) is the basic compatibility condition of (5.1) To determine $p(r, z)$, the r. h. s. of (5.3), (5.4) may be outlined in terms of $A_{\varphi}$ and $Q^{\prime \prime}$ :

$$
\nabla p=2 \frac{\mathrm{d} \eta}{\mathrm{d} z} \cdot \nabla\left(\frac{1}{r} \frac{\partial}{\partial r}\left(r A_{\varphi}\right)\right)+\nabla \times\left(\hat{\mathbf{e}}_{\varphi} Q^{\prime \prime}\right) .
$$

Taking the divergence, we arrive at an inhomogeneous potential equation of $p(r, z)$ :

$$
\Delta p=\nabla \cdot\left[2 \frac{\mathrm{d} \eta}{\mathrm{d} z} \cdot \nabla\left(\frac{1}{r} \frac{\partial}{\partial r}\left(r A_{\varphi}\right)\right)\right] .
$$

For $\eta(z)=$ const , we have $\Delta p=0$ as also concluded from (2.9). If any solution $Q^{\prime \prime}(r, z), A_{\varphi}(r, z)$ of $(2.21),(2.22)$ is substituted on the r. h. s. of (5.6), the latter equation becomes a compatible one, and $p(r, z)$ can be calculated, 
up to the additive constant $p_{0}$, by the integration of (5.6) along an arbitrary path. We choose the radial one. We introduce $Q(r, z)$ according to

$\partial Q(r, z) / \partial r=-Q^{\prime \prime}(r, z)$

$Q(r, z)$ is uniquely determined by (5.8) up to a $z$-dependent function $g(z)$. The latter must satisfy the homogeneous part of (5.7), therefore $g(z)=p_{0} z$ with arbitrary $p_{0}$. Thus, we arrive at (2.24).

\section{Appendix 2: AMSA treatment of RBC}

We will satisfy the RBC based on a superposition of functions $\overline{\bar{Q}}_{i}^{\prime \prime}(r, Z), \overline{\bar{A}}_{\varphi i}(r, Z), i=0,1,2, \ldots$, to be successively determined. Each function pair $\overline{\bar{Q}}_{i}^{\prime \prime}, \overline{\bar{A}}_{\varphi i}$ may be a solution of (3.7), (3.8) and represented analogously to (3.10), (3.11), adding the lower index $i$ on the r.h.s. In the same way, the hydrodynamic functions and the force components derived from $\overline{\bar{Q}}_{i}^{\prime \prime}, \overline{\bar{A}}_{\varphi i}$ may be represented analogously to (3.12) to (3.14), and (3.17), (3.18), respectively, adding the lower index $i$ on both sides.

At the start, let us assume a lowest order solution $\varepsilon \overline{\bar{Q}}_{0}^{\prime \prime(1)}(r, Z),(1 / \varepsilon) \cdot \overline{\bar{A}}_{\varphi 0}^{(-1)}(r, Z)$, and $\overline{\bar{p}}_{0}^{(0)}(r, Z)$, which satisfies (3.7), (3.8), and (3.9) up to $\varepsilon^{3}, \varepsilon$, and $\varepsilon^{2}$, respectively. The former may involve any $Z$-dependent coefficients to satisfy the normal force balance (3.20) in $\varepsilon^{0}$. After substitution on the 1.h.s. of (2.13), the normal force balance (3.20) may be written as

$\overline{\bar{F}}_{\text {norm } 0}^{(0)}(R, Z)= \pm 2 \overline{\bar{H}}^{(0)}(R, Z) / \eta(Z)$.

Next, $\varepsilon \overline{\bar{Q}}_{0}^{\prime \prime(1)},(1 / \varepsilon) \overline{\bar{A}}_{\varphi 0}^{(-1)}$, and $\overline{\bar{p}}_{0}^{(1)}$ will be taken as the base to derive the complete solution of (3.7) to (3.9) as perturbation series according to $\overline{\bar{Q}}_{0}^{\prime \prime}(r, Z, \varepsilon)=\varepsilon \overline{\bar{Q}}_{0}^{\prime \prime(1)}(r, Z)+\varepsilon^{3} \overline{\bar{Q}}_{0}^{\prime \prime(3)}(r, Z)+\cdots, \overline{\bar{A}}_{\varphi 0}(r, Z, \varepsilon)=(1 / \varepsilon) \overline{\bar{A}}_{\varphi 0}^{(-1)}(r, Z)+$ $\varepsilon \overline{\bar{A}}_{\varphi 0}^{(1)}(r, Z)+\cdots$, and $\overline{\bar{p}}_{0}(r, Z, \varepsilon)=\overline{\bar{p}}_{0}^{(0)}(r, Z)+\varepsilon^{2} \overline{\bar{p}}_{0}^{(2)}(r, Z)+\cdots$. Substitution of $\overline{\bar{Q}}_{0}^{\prime \prime}, \overline{\bar{A}}_{\varphi 0}$, and $\overline{\bar{p}}_{0}$ on the 1.h.s. of (2.13), (2.14) will specify (3.15), (3.16) to $\overline{\bar{F}}_{\text {norm } 0}(R, Z, \varepsilon)=\overline{\bar{F}}_{\text {norm } 0}^{(0)}(R, Z)+\varepsilon^{2} \overline{\bar{F}}_{\text {norm } 0}^{(2)}(R, Z)+\cdots$, $\overline{\bar{F}}_{\tan 0}(R, Z, \varepsilon)=\varepsilon \overline{\bar{F}}_{\tan 0}^{(1)}(R, Z)+\varepsilon^{3} \overline{\bar{F}}_{\tan 0}^{(3)}(R, Z)+\cdots$.

Obviously, $\overline{\bar{Q}}_{0}^{\prime \prime}, \overline{\bar{A}}_{\varphi 0}$, and $\overline{\bar{p}}_{0}$ induce redundant higher-order force contributions $\varepsilon \overline{\bar{F}}_{\tan 0}^{(1)}(R, Z), \varepsilon^{2} \overline{\bar{F}}_{\text {norm } 0}^{(2)}(R, Z)$, $\ldots$, which are not balanced in $\varepsilon^{0}$. To satisfy (3.21) in $\varepsilon^{1}$, we have to look for novel AMSA solutions $\overline{\bar{Q}}_{1}^{\prime \prime}(r, Z, \varepsilon)$, $\overline{\bar{A}}_{\varphi 1}(r, Z, \varepsilon)$, and $\overline{\bar{p}}_{1}(r, Z, \varepsilon)$ of (3.7) to (3.9) such that a normal force contribution in $\varepsilon^{0}$ does not appear, and

$\varepsilon \overline{\bar{F}}_{\tan 1}(R, Z, \varepsilon)+\varepsilon \overline{\bar{F}}_{\tan 0}^{(1)}(R, Z)=0$,

holds true in $\varepsilon^{1} . \varepsilon \overline{\bar{F}}_{\tan 1}(R, Z, \varepsilon)=\varepsilon \overline{\bar{F}}_{\tan 1}^{(1)}(R, Z)+\varepsilon^{3} \overline{\bar{F}}_{\tan 1}^{(3)}(R, Z)+\cdots$ is calculated from the 1.h.s. of (2.14) after substitution of $\overline{\bar{Q}}_{1}^{\prime \prime}, \overline{\bar{A}}_{\varphi 1}$, and $\overline{\bar{p}}_{1}$. Like above, the latter functions induce redundant second- and higher-order contributions to $\overline{\bar{F}}_{\text {norm }}(R, Z, \varepsilon)$ and $\overline{\bar{F}}_{\tan }(R, Z, \varepsilon)$. This requires novel solutions $\overline{\bar{Q}}_{2}^{\prime \prime}, \overline{\bar{A}}_{\varphi 2}$, and $\overline{\bar{p}}_{2}$ of (3.7) to (3.9) to satisfy the normal force balance in $\varepsilon^{2}$ according to

$\varepsilon^{2} \overline{\bar{F}}_{\text {norm } 2}(R, Z, \varepsilon)+\varepsilon^{2} \overline{\bar{F}}_{\text {norm } 2}^{(2)}(R, Z)= \pm \varepsilon^{2} \cdot 2 \overline{\bar{H}}^{(2)}(R, Z) / \eta(Z)$ 
where $\varepsilon^{2} \overline{\bar{F}}_{\text {norm2 }}(R, Z, \varepsilon)=\varepsilon^{2} \overline{\bar{F}}_{\text {norm2 }}^{(2)}(R, Z)+\varepsilon^{4} \overline{\bar{F}}_{\text {norm2 }}^{(4)}(R, Z)+\cdots$ is calculated from the 1.h.s. of (2.13) after substitution of $\overline{\bar{Q}}_{2}^{\prime \prime}, \overline{\bar{A}}_{\varphi 2}$, and $\overline{\bar{p}}_{2}$, and so on.

\section{Appendix 3: AMSA in the zeroth and first orders}

Zeroth order

We want to determine $\overline{\bar{Q}}_{0}^{\prime \prime}, \overline{\bar{A}}_{\varphi 0}$, and $\overline{\bar{p}}_{0}$ to reproduce the results of Sect. 3.1 with $Z$-dependent coefficients. The normal force balance in $\varepsilon^{0}$ to be satisfied on both the boundaries requires two independent pairs of solutions of (3.7) to (3.9). We look for ${ }^{(s)} \overline{\bar{Q}}_{0}^{\prime \prime},{ }^{(s)} \overline{\bar{A}}_{\varphi 0}$, and ${ }^{(r)} \overline{\bar{Q}}_{0}^{\prime \prime},{ }^{(r)} \overline{\bar{A}}_{\varphi 0}$, with singular and regular behavior for $r \rightarrow 0$, denoted by the upper index $(s)$ and $(r)$, respectively.

The perturbation series of ${ }^{(s)} \overline{\bar{Q}}_{0}^{\prime \prime},{ }^{(s)} \overline{\bar{A}}_{\varphi 0}$ start with $\varepsilon \cdot{ }^{(s)} \overline{\bar{Q}}_{0}^{\prime \prime(1)},(1 / \varepsilon) \cdot(s) \overline{\bar{A}}_{\varphi 0}^{(-1)}$, where

${ }^{(s)} \overline{\bar{Q}}_{0}^{\prime \prime(1)}(r, Z)=\eta(Z) \cdot(1 / r) \cdot \mathrm{d} C_{A 0}(Z) / \mathrm{d} Z$,

$(s) \overline{\bar{A}}_{\varphi 0}^{(-1)}(r, Z)=(1 / r) \cdot \int_{0}^{Z} \mathrm{~d} Z_{1} C_{A 0}\left(Z_{1}\right)$,

and $C_{A 0}(Z)$ is determined below. To find ${ }^{(r)} \overline{\bar{Q}}_{0}^{\prime \prime}$ and ${ }^{(r)} \overline{\bar{A}}_{\varphi 0}$, the constant pressure ansatz of Sect. 3.1 has to be generalized in AMSA to

$\overline{\bar{p}}_{0}^{(0)}(r, Z)=C_{P 0}(Z)$,

now describing a pressure field with axial gradient. The latter has to be considered as the driving force of that flow field contribution derived from ${ }^{(r)} \overline{\bar{Q}}_{0}^{\prime \prime}$, and ${ }^{(r)} \overline{\bar{A}}_{\varphi 0}$. We find

$\begin{aligned}{ }^{(r)} \overline{\bar{Q}}_{0}^{\prime \prime}(z, Z, \varepsilon) & =(\varepsilon / 2) r \mathrm{~d} C_{P 0}(Z) / \mathrm{d} Z+O\left(\varepsilon^{3}\right), \\ { }^{(r)} \overline{\bar{A}}_{\varphi 0}(z, Z, \varepsilon) & =(\varepsilon / 16) r^{3}\left(\mathrm{~d} C_{P 0}(Z) / \mathrm{d} Z\right) \cdot(1 / \eta(Z))+O\left(\varepsilon^{3}\right) .\end{aligned}$

Equation (5.14) is the starting term of (3.12) and $C_{P 0}(Z)$ is determined below.

The lowest order term of (3.17) derived from (5.12) and (5.13), and that from (5.15) and (5.16) are

$(s) \overline{\bar{F}}_{\text {norm } 0}^{(0)}(R, Z)=-2 C_{A 0}(Z) / R^{2}$

and

${ }^{(r)} \overline{\bar{F}}_{\text {norm0 }}^{(0)}(R, Z)=-C_{P 0}(Z) / \eta(Z)$,

respectively. Because of $\overline{\bar{F}}_{\text {norm0 }}^{(0)}(r, Z)={ }^{(r)} \overline{\bar{F}}_{\text {norm } 0}^{(0)}(r, Z)+{ }^{(s)} \overline{\bar{F}}_{\text {norm } 0}^{(0)}(r, Z)$, the zeroth-order RBC (5.9) now read $\overline{\bar{F}}_{\text {norm } 0}^{(0)}(R, Z)= \pm((1 / \eta(Z)) \cdot(1 / R)$,

taking the lower sign for $R=R_{1}(Z)$. (5.19) determines $C_{A 0}(Z), C_{P 0}(Z)$ according to

$C_{A 0}(Z)=(1 / 2 \eta(Z)) \cdot R_{0}(Z) R_{1}(Z) /\left(R_{0}(Z)-R_{1}(Z)\right)$,

$C_{P 0}(Z)=1 /\left(R_{0}(Z)-R_{1}(Z)\right)$. 
First order

We follow the schema outlined in Sect. 3.3 and look for functions $\overline{\bar{Q}}_{1}^{\prime \prime}(r, Z, \varepsilon)$ and $\overline{\bar{A}}_{\varphi 1}(r, Z, \varepsilon)$ required, according to (5.10), to balance the redundant first-order tangential force contribution $\varepsilon \overline{\bar{F}}_{\tan 0}^{(1)}(R, Z)$ from the zeroth-order AMSA. From (3.13), (3.14), (3.16), (3.18), and (5.13) to (5.16), we find

$\varepsilon \overline{\bar{F}}_{\tan 0}^{(1)}(R, Z)=\varepsilon\left(\mathrm{d} C_{A 0}(Z) / \mathrm{d} Z\right) / R-(\varepsilon / 2 \eta(Z))\left(\mathrm{d} C_{P 0}(Z) / \mathrm{d} Z\right) \cdot R-2 \varepsilon(\mathrm{d} R / \mathrm{d} Z) C_{A 0}(Z) / R^{2}$,

where $R, \mathrm{~d} R / \mathrm{d} Z$ stands for $R_{0}, \mathrm{~d} R_{0} / \mathrm{d} Z$, and $R_{1}, \mathrm{~d} R_{1} / \mathrm{d} Z$. To satisfy (5.10) on both the boundaries, $\overline{\bar{Q}}_{1}^{\prime \prime}$ and $\overline{\bar{A}}_{\varphi 1}$ must be the sum of two independent solutions ${ }^{(s)} \overline{\bar{Q}}_{1}^{\prime \prime}$ and ${ }^{(s)} \overline{\bar{A}}_{\varphi 1}$, and ${ }^{(r)} \overline{\bar{Q}}_{1}^{\prime \prime}$ and ${ }^{(r)} \overline{\bar{A}}_{\varphi 1}$ of (3.7), (3.8) with singular and regular behavior for $r \rightarrow 0$ (upper index $(s)$ and $(r)$ ), respectively, which altogether contribute to the first-order tangential force $\varepsilon \overline{\bar{F}}_{\tan 1}^{(1)}(R, Z)=-\varepsilon \overline{\bar{\sigma}}_{r z 1}^{\prime(1)}(R, Z)$. These novel solutions read

$$
\begin{aligned}
{ }^{(s)} \overline{\bar{Q}}_{1}^{\prime \prime}(r, Z, \varepsilon) & =\varepsilon \cdot(1 / r) \cdot C_{A s}^{(1)}(Z) / \eta(Z)+O\left(\varepsilon^{3}\right), \\
{ }^{(s)} \overline{\bar{A}}_{\varphi 1}(r, Z, \varepsilon) & =\varepsilon \cdot(1 / 2) \cdot r \cdot \log r \cdot C_{A s}^{(1)}(Z)+O\left(\varepsilon^{3}\right), \\
{ }^{(r)} \overline{\bar{Q}}_{1}^{\prime \prime}(r, Z, \varepsilon) & =\varepsilon \cdot r \cdot C_{A r}^{(1)}(Z) / \eta(Z)+O\left(\varepsilon^{3}\right), \\
{ }^{(r)} \overline{\bar{A}}_{\varphi 1}(r, Z, \varepsilon) & =\varepsilon \cdot\left(r^{3} / 8\right) \cdot C_{A r}^{(1)}(Z)+O\left(\varepsilon^{3}\right),
\end{aligned}
$$

with two arbitrary functions $C_{A s}^{(1)}(Z), C_{A r}^{(1)}(Z)$ to be determined from (5.10). The result is

$$
\begin{aligned}
& C_{A s}^{(1)}(Z)=2 C_{A 0}(Z) \frac{R_{1}(Z)^{3} \cdot \mathrm{d} R_{0}(Z) / \mathrm{d} Z-R_{0}(Z)^{3} \cdot \mathrm{d} R_{1}(Z) / \mathrm{d} Z}{R_{0}(Z) R_{1}(Z)\left(R_{0}(Z)^{2}-R_{1}(Z)^{2}\right)}+\frac{\mathrm{d} C_{A 0}(Z)}{\mathrm{d} Z}, \\
& C_{A r}^{(1)}(Z)=2 C_{A 0}(Z) \frac{R_{1}(Z) \cdot \mathrm{d} R_{0}(Z) / \mathrm{d} Z-R_{0}(Z) \cdot \mathrm{d} R_{1}(Z) / \mathrm{d} Z}{R_{0}(Z) R_{1}(Z)\left(R_{0}(Z)^{2}-R_{1}(Z)^{2}\right)} .
\end{aligned}
$$

Equations (5.23) to (5.28) is the base to calculate all hydrodynamic quantities within the first perturbation order. These first-order corrections depend linearly on $\mathrm{d} R_{0}(Z) / \mathrm{d} Z, \mathrm{~d} R_{1}(Z) / \mathrm{d} Z$, and $(\mathrm{d} / \mathrm{d} Z)(1 / \eta(Z))$. For non-inclined boundaries, we have $C_{A r}^{(1)}(Z)=0$ and $C_{A s}^{(1)}(Z) \propto(\mathrm{d} / \mathrm{d} Z)(1 / \eta(Z))$. Continuing to higher-order approaches, crossover effects will be included, and we rapidly arrive at very complicated expressions including higher than first-order derivatives of $R_{0}(Z)$ and $R_{1}(Z)$.

\section{Appendix 4: First-order corrections of geometry parameters}

To determine $R_{01}(Z)$ and $W_{1}(Z)$ of (3.47), (3.48), we substitute (3.25), (3.26) into (3.31), (3.32), subtract both the equations, and expand the r.h.s. in powers of $W(Z) / R_{0}(Z)$ (which starts with the negative power $\left(W / R_{0}\right)^{-3}$ ). Observing (3.47) and (3.48), the comparison in $\varepsilon^{1}$ yields

$$
\begin{aligned}
& \frac{\mathrm{d} W_{1}(Z)}{\mathrm{d} Z}=\frac{1}{\eta(Z)}\left(\frac{\mathrm{d}}{\mathrm{d} Z} \frac{1}{\eta(Z)}\right) \frac{1}{u^{2}}{ }^{(\eta)} K\left(R_{00}(Z), W_{0}(Z)\right)+\frac{1}{\eta(Z)^{3}} \cdot \frac{1}{u^{3}}{ }^{(\mathrm{kin})} K\left(R_{00}(Z), W_{0}(Z)\right), \\
& { }^{(\eta)} K\left(R_{00}(Z), W_{0}(Z)\right)=\frac{1}{8} \frac{R_{00}(Z)}{W_{0}(Z)} \cdot R_{00}(Z)\left(1-2 \log R_{00}(Z)\right)+(1 / 4) R_{00}(Z) \cdot \log R_{00}(Z)+\cdots, \\
& { }^{(\operatorname{kin})} K\left(R_{00}(Z), W_{0}(Z)\right)=-\frac{R_{00}(Z)^{3}}{8 W_{0}(Z)^{3}}+\frac{R_{00}(Z)^{2}}{8 W_{0}(Z)^{2}}\left(2-\log R_{00}(Z)\right)+\cdots
\end{aligned}
$$


For further evaluation, we subdivide $W_{0}(Z)=W_{0}(0)-(1 / 2 u) \cdot \int_{0}^{Z} \mathrm{~d} Z_{1}\left(1 / \eta\left(Z_{1}\right)\right)$ according to (3.37), (3.38), and apply convergent series expansions of $R_{00}(Z)$ and $R_{00}(Z) / W_{0}(Z)$ in powers of $(1 / 2 u) \cdot \int_{0}^{Z} \mathrm{~d} Z_{1}\left(1 / \eta\left(Z_{1}\right)\right) / W_{0}(Z)$, derived from (3.42). We get

$$
\begin{aligned}
& R_{00}(Z)=\frac{1-R_{1 \max }^{2}+W_{0}(0)^{2}}{2 W_{0}(0)}+\frac{1}{2 u} \int_{0}^{Z} \frac{\mathrm{d} Z_{1}}{\eta\left(Z_{1}\right)} \cdot \frac{1-R_{1 \max }^{2}+W_{0}(0)^{2}}{2 W_{0}(0)^{2}}+\cdots, \\
& \frac{R_{00}(Z)}{W_{0}(Z)}=\frac{1-R_{1 \max }^{2}+W_{0}(0)^{2}}{2 W_{0}(0)^{2}}+\frac{1}{2 u} \int_{0}^{Z} \frac{\mathrm{d} Z_{1}}{\eta\left(Z_{1}\right)} \cdot \frac{1-R_{1 \max }^{2}}{W_{0}(0)^{2}}+\cdots
\end{aligned}
$$

It should be pointed out that due to the $u$-dependence of $W_{0}(0)$, the order of subsequent terms of (5.32), (5.33) decreases like powers of $\Delta Z_{H} /\left(4 u\left(1-R_{1 \min }\right)+\Delta Z_{H}\right)$.

If $u$ undergoes the restriction (in addition to (3.34))

$u>\Delta Z_{H} / 4\left(1-R_{1 \max }\right)$,

(5.32), (5.33) can be rearranged into more rapidly converging series expansions in powers of $1 / u$ so that $R_{00}(Z)=$ $1+O(1 / u), R_{00}(Z) / W_{0}(Z)=1 /\left(1-R_{1 \max }\right)+O(1 / u)$. In the same way, (5.29) to (5.31) can be rearranged into series expansions in powers of $1 / u$.

The first-order correction $\Delta W_{1}^{(\text {tot })}$ to $\Delta W^{(\text {tot })}$ is

$\Delta W_{1}^{(\mathrm{tot})}=-\int_{-\infty}^{\infty} \mathrm{d} W_{1}(Z) / \mathrm{d} Z$

We will focus on the main contribution in $1 / u$ of $\Delta W_{1}^{(\text {tot })}$, if (3.34) and (5.34) hold true. Evaluating through (5.35), (5.29) to (5.31), the leading order $\propto 1 / u^{2}$ comes from the non-kinematic axial flow only. This contribution cancels because of $\lim _{|Z| \rightarrow \infty}(1 / \eta(Z))=0$. This is obviously explained through the alternating direction of the non-kinematic axial flow across the collapsing zone, according to (3.26).

\section{References}

1. Lewis JA (1977) The collapse of a viscous tube. J Fluid Mech 81:129-135

2. Kirchhof J (1980) A hydrodynamic theory of the collapsing process for the preparation of optical waveguide preforms. Phys Stat Sol (a) 60:K127-K131

3. Kirchhof J (1985) Reactor problems in modified chemical vapour deposition (I). The collapse of quartz tubes. Cryst Res Technol 20:705-712

4. Kirchhof J, Funke A (1986) Reactor problems in modified chemical vapour deposition (II). The mean viscosity of quartz glass reactor tubes. Cryst Res Technol 21:763-770

5. Geyling FT, Walker KL, Csencsits R (1983) The viscous collapse of thick-walled tubes. J Appl Mech 50:303-310

6. Das SK, Gandhi KS (1986) A model for thermal collapse of tubes. Application for optical glass fibres. Chem Eng Sci 41:73-81

7. Yarin AL, Bernat V, Doupovec J, Miklos P (1993) The viscous collapse of radial nonsymmetric composite tubes. J Lightwave Technol 11:198-204

8. Makovetskii AA, Zamyatin AA, Ivanov GA (2014) Technique for estimating the viscosity of molten silica glass on the kinetics of the collapse of the glass capillary. Glass Phys Chem 40:526-530

9. Fitt AD, Furusawa K, Monro TM, Please CP, Richardson DJ (2002) The mathematical modeling of capillary drawing for holey fibre manufacture. J Eng Math 43:201-227

10. Geyling FT (1976) Basic fluid dynamic considerations in the drawing of optical fibers. Bell Syst Tech J 55:1011-1056

11. Knight JC, Birks TA, Cregan RE, Russell PStJ, de Sandro J-P (1999) Photonic crystals as optical fibres-physics and applications. Opt Mater 11:143-151

12. Knight JC (2003) Photonic crystal fibres. Nature 424:847-851

13. Snyder AW, Love JD (1993) Optical waveguide theory. Chapman and Hall, London

14. Peak UC, Schroeder CM, Kurkjian CM (1988) Determination of the viscosity of high silica glasses during fiber drawing. Glass Technol 29:263-266 
15. Peak UC, Bunk RB (1978) Physical behavior of the neck-down region during furnace drawing of silica fibers. J Appl Phys 48:4417-4422

16. Geyling FT, Homsy GM (1980) Extensional instabilities of the glass fibre drawing process. Glass Technol 21:95-102

17. Schultz WW, Davis SH (1982) One-dimensional liquid fibres. J Rheol 26:331-345

18. Huynh BP, Tanner RI (1983) Study of non-isothermal glass fibre drawing process. Rheol Acta 22:482-499

19. Rosenberg SE, Papamichael H, Miaoulis IN (1994) A two-dimensional analysis of the viscous problem of a glass preform during the optical fibre drawing process. Glass Technol 35:260-264

20. Sarboh SD, Milinkovic SA, Debeljkovic DLJ (1998) Mathematical model of the glass capillary tube drawing process. Glass Technol 39:53-67

21. Yin Z, Jaluria Y (1998) Thermal transport and flow in high-speed optical fiber drawing. J Heat Transf 120:916-930

22. Fitt AD, Furusawa K, Monro TM, Please CP (2001) Modelling of the fabrication of hollow fibers: capillary drawing. J Lightwave Technol 19:1924-1931

23. Yan Q, Yan Q (2004) Numerical simulation of the viscosity flow process in the region of formation of basalt fibre. Glass Technol 45:227-232

24. Griffiths IM, Howell PD (2007) The surface-tension driven evaluation of a two-dimensional annular viscous tube. J Fluid Mech 593:181-208

25. Voyce CJ, Fitt AD, Monro TM (2008) Mathematical modeling as an accurate predictive tool in capillary and microstructured fiber manufacture: The effect of preform rotation. J Lightwave Technol 26:791-798

26. Voyce CJ, Fitt AD, Hayes RJ, Monro TM (2009) Mathematical modeling of the self-pressuring mechanism for microstructured fiber drawing. J Lightwave Technol 27:871-878

27. Luzi G, Epple P, Scharrer M, Fujimoto K, Rauh C, Delgado A (2010) Influence of surface tension and inner pressure on the process of fiber drawing. J Lightwave Technol 28(2010):1882-1888

28. Luzi G, Epple P, Scharrer M, Fujimoto K, Rauh C, Delgado A (2011) Comparison between an analytical asymptotic fiber drawing model with full Navier-Stokes solution taking into account the effects of inner pressure and surface tension. J Lightwave Technol 29:1638-1646

29. Kostecki R, Ebendorff-Heidepriem H, Warren-Smith SC, Monro TM (2014) Predicting the drawing conditions for microstructured optical fiber fabrication. Opt Mater Express 4:29-40

30. Rayleigh L (1892) On the instability of a cylinder of viscous liquid under capillary force. Lond, Edinb, Dublin Philos Mag J Sci $34: 145-154$

31. Matovich MR, Pearson R (1969) Spinning a molten threadline-steady state isothermal viscous flows. Ind Eng Chem Fundam 8:512-520

32. Dewynne JN, Ockendon JR, Wilmott P (1992) A systematic derivation of the leading-order equations for extensional flows in slender geometries. J Fluid Mech 244:323-338

33. Stokes YM, Buchak P, Crowdy DG, Ebendorff-Heidepriem H (2014) Drawing of microstructured fibres: circular and non-circular tubes. J Fluid Mech 755:176-203

34. Buchak P, Crowdy DC, Stokes YM, Ebendorff-Heidepriem H (2015) Elliptical pore regularization of the inverse problem of microstructured optical fibre fabrication. J Fluid Mech 778:5-38

35. Chen MJ, Stokes YM, Buchak P, Crowdy DC, Ebendorff-Heidepriem H (2015) Microstructured optical fibre drawing with active channel pressurization. J Fluid Mech 783:137-165

36. Modest MF (2013) Radiative heat transfer, 3rd edn. Academic Press, Oxford

37. Hollandt J, Friedrich R, Gutschwager B, Taubert D, Hartmann J (2004) High- accuracy radiation thermometry at the National Metrology Institute of Germany, the PTB. High Temp High Press 35(36):379-415

38. Bernhardt F (Ed.) (2004) Technische Temperaturmessung. Springer, Berlin

39. Scholze H (1990) Glass: nature, structure, and properties. Springer, New York

40. Kirchhof J, Unger S (2017) Viscous behavior of synthetic silica glass tubes during collapsing. Optical Materials Express 7:386-400

41. Landau LD, Lifshitz EM (1966) Hydrodynamik. Akademie, Berlin

42. Sommerfeld A (1970) Vorlesungen über theoretische Physik Band II: Mechanik der deformierbaren Medien. Akademische Verlagsgesellschaft Geest und Portig, Leipzig

43. Baule B (1955) Die Mathematik des Naturforschers und Ingenieurs, Band VII: Differentialgeometrie. S. Hirzel, Leipzig

44. Nayfeh AH (1981) Introduction to perturbation techniques. Wiley, New York

45. Adomian G (1988) A review of the decomposition method in applied mathematics. J Math Anal Appl 135:501-544 